\title{
PubChem atom environments
}

\author{
Volker D Hähnke, Evan E Bolton* and Stephen H Bryant
}

\begin{abstract}
Background: Atom environments and fragments find wide-spread use in chemical information and cheminformatics. They are the basis of prediction models, an integral part in similarity searching, and employed in structure search techniques. Most of these methods were developed and evaluated on the relatively small sets of chemical structures available at the time. An analysis of fragment distributions representative of most known chemical structures was published in the 1970s using the Chemical Abstracts Service data system. More recently, advances in automated synthesis of chemicals allow millions of chemicals to be synthesized by a single organization. In addition, open chemical databases are readily available containing tens of millions of chemical structures from a multitude of data sources, including chemical vendors, patents, and the scientific literature, making it possible for scientists to readily access most known chemical structures. With this availability of information, one can now address interesting questions, such as: what chemical fragments are known today? How do these fragments compare to earlier studies? How unique are chemical fragments found in chemical structures?
\end{abstract}

Results: For our analysis, after hydrogen suppression, atoms were characterized by atomic number, formal charge, implicit hydrogen count, explicit degree (number of neighbors), valence (bond order sum), and aromaticity. Bonds were differentiated as single, double, triple or aromatic bonds. Atom environments were created in a circular manner focused on a central atom with radii from 0 (atom types) up to 3 (representative of ECFP_6 fragments). In total, combining atom types and atom environments that include up to three spheres of nearest neighbors, our investigation identified 28,462,319 unique fragments in the 46 million structures found in the PubChem Compound database as of January 2013. We could identify several factors inflating the number of environments involving transition metals, with many seemingly due to erroneous interpretation of structures from patent data. Compared to fragmentation statistics published 40 years ago, the exponential growth in chemistry is mirrored in a nearly eightfold increase in the number of unique chemical fragments; however, this result is clearly an upper bound estimate as earlier studies employed structure sampling approaches and this study shows that a relatively high rate of atom fragments are found in only a single chemical structure (singletons). In addition, the percentage of singletons grows as the size of the chemical fragment is increased.

Conclusions: The observed growth of the numbers of unique fragments over time suggests that many chemically possible connections of atom types to larger fragments have yet to be explored by chemists. A dramatic drop in the relative rate of increase of atom environments from smaller to larger fragments shows that larger fragments mainly consist of diverse combinations of a limited subset of smaller fragments. This is further supported by the observed concomitant increase of singleton atom environments. Combined, these findings suggest that there is considerable opportunity for chemists to combine known fragments to novel chemical compounds. The comparison of PubChem to an older study of known chemical structures shows noticeable differences. The changes suggest advances in synthetic capabilities of chemists to combine atoms in new patterns. Log-log plots of fragment incidence show small numbers of fragments are found in many structures and that large numbers of fragments are found in very few structures, with nearly half being novel using the methods in this work. The relative decrease in the count of new

\footnotetext{
*Correspondence: bolton@ncbi.nlm.nih.gov

Department of Health and Human Services, National Center

for Biotechnology Information, National Library of Medicine, National

Institutes of Health, 8600 Rockville Pike, Bethesda, MD 20894, USA
}

Chemistry Central
C 2015 Hähnke et al. This article is distributed under the terms of the Creative Commons Attribution 4.0 International License (http://creativecommons.org/licenses/by/4.0/), which permits unrestricted use, distribution, and reproduction in any medium, provided you give appropriate credit to the original author(s) and the source, provide a link to the Creative Commons license, and indicate if changes were made. The Creative Commons Public Domain Dedication waiver (http://creativecommons.org/ publicdomain/zero/1.0/) applies to the data made available in this article, unless otherwise stated. 
fragments as a function of size further suggests considerable opportunity for more novel chemicals exists. Lastly, the differences in atom environment diversity between PubChem Substance and Compound showcase the effect of PubChem standardization protocols, but also indicate that a normalization procedure for atom types, functional groups, and tautomeric/resonance forms based on atom environments is possible. The complete sets of atom types and atom environments are supplied as supporting information.

Keywords: Molecular graph, PubChem, Fragment, Standardization, SMARTS

\section{Background}

The de facto standard for the representation of small molecules in chemical information and cheminformatics is the molecular graph, a mathematical construct providing the topological description of a chemical structure as a set of vertices (corresponding to atoms), and edges between those vertices (corresponding to bonds between atoms) [1, 2]. The molecular graph is deeply rooted in valence bond theory, where the structure diagram is (essentially) equivalent to the Lewis structure of a molecule $[3,4]$. It helps provide the basis for several related chemical descriptions: systematic names [5-7], line notations [8-22], and connection table-based file formats [23-26]. The valence bond model description of a chemical structure has proven to be incredibly useful to chemists, even though it is simplistic compared to a full quantum mechanical description. Subgraphs, referred to as substructures or molecular fragments, are the key concept in a variety of standard methods for the assessment of chemical similarity [27-34], clustering [35-39], and structure searching [40-42]. For example, fragmentbased approaches of atom-centered or variable topological characteristics are used to accelerate chemical structure searches in databases [43-45].

Chemical fragments are interesting in that they can have many uses. More than 40 years ago an atom-centered fragmentation model, referred to as 'augmented atoms', was used to analyze 28,799 molecules sampled from the Chemical Abstracts Service Registry System (CASRS) [46, 47]. In that study, 2,331 unique atom environments could be distinguished based on the most discriminative of the employed fragment models, which considered atomic number and seven bond types (single/ double/triple bond in a chain or ring, respectively; and aromatic bond in a ring). The resulting top-10 ranked fragments and their incidences (count of structures containing a fragment) and occurrences (count of all cases of a fragment across all structures) from this analysis are reproduced in Fig. 1. One can consider this set of 2,331 augmented atoms a representation of the ability of chemists at that point in time to synthesize and isolate novel chemical substances (with every sampled chemical known to the CASRS at that point in time being some combination of these 2,331 fragments). If the count of scientific publications [48] and known chemical substances $[49,50]$ illustrated in Fig. 2 are any indication, chemistry as a science has progressed significantly in 40 years. But how have chemists progressed in terms of their ability to synthesize and isolate novel chemistry between then and now? Using this earlier study of CASRS, one could rephrase this question as: what chemical fragments exist in chemical structures today that did not exist then?

In the world of chemical information much has changed since the 1970s. For example, aided by

\begin{tabular}{|c|c|c|c|c|c|c|}
\hline & \\
Occurrence \\
Incidence
\end{tabular}



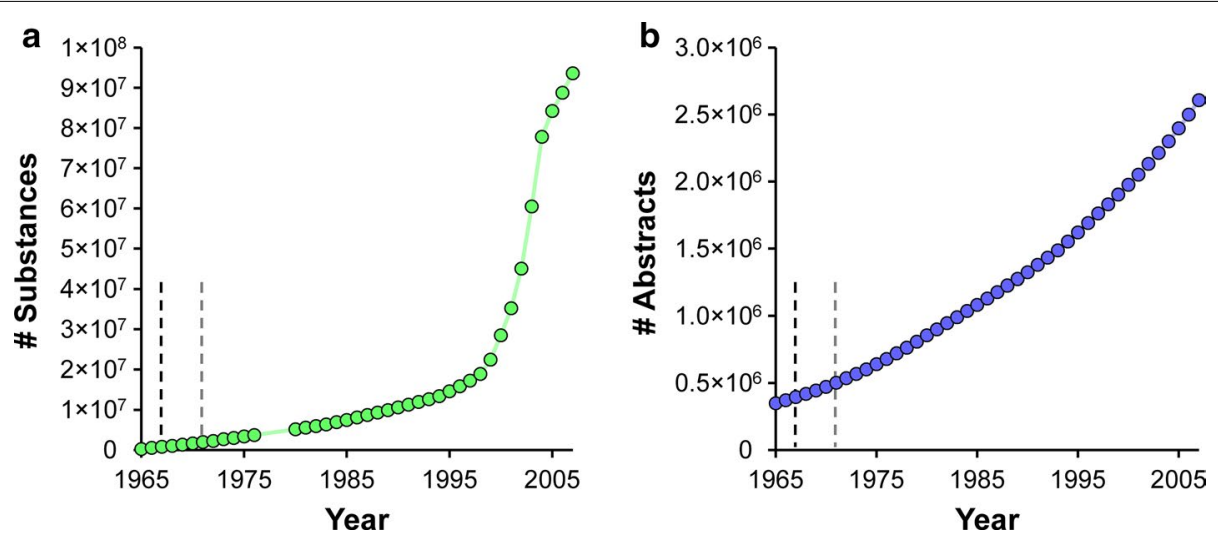

Fig. 2 Exponential growth in chemistry. Data reflects registered $\mathbf{a}$ substances; and $\mathbf{b}$ abstracts on record at Chemical Abstracts Service at year end [50]. No substances data is available for years 1977-1979. Black dashed line indicates time of random sampling of 28,963 structures from the 593,071 individual structures in the Chemical Abstracts Registry System [46]. Gray dashed line indicates time of the 'augmented atom' analysis by Adamson et al. on this subset [47]. Numbers for abstracts on record include papers, patents and books.

computers and the internet, chemical information data exchange has become increasingly open. There are now chemical data repositories providing access to large quantities of aggregated chemical information without barriers or paywalls. An example of one of these repositories is PubChem.

PubChem is an open archive for chemical substances and their biological activities [51-54]. It consists of three distinct primary databases: Substance, Compound and BioAssay. Substance contains descriptions of chemical substances as provided by hundreds of contributors. BioAssay contains bioactivity information about chemical substances. Compound is derived from entries in Substance via automated protocols that generate a preferred chemical representation and identify equivalent chemicals between PubChem contributors. At the time of initially writing this manuscript (January 2013), PubChem contained more than 100 million substance and 46 million compound records. Given the size and breadth of contributing organizations (including many substance suppliers, patent databases, natural product collections, literature databases, etc.), PubChem might be considered to represent a rather large fraction of all known (small molecule, organic) chemistry.

Using PubChem chemical contents and the earlier analysis method used with CASRS, this study assesses the overall progress by chemists to access novel chemistry over the past 40 years as a function of new, unique chemical fragments. In addition, we present here detailed statistics about atom environments of different sizes in the PubChem Substance (non-standardized structures as provided by contributors) and PubChem Compound (standardized unique structures) databases.

\section{Results and discussion}

\section{Terminology and approach}

Unless stated otherwise, the following approach and definitions were used for the purpose of this study. Incidence refers to the absolute count or percentage of (substance or compound) records that contain a particular fragment. Occurrence refers to the absolute count or percentage of all fragments across all structures (substance or compound) considered. Atom environments are defined as circular atom-centered topological neighborhood fragments of varying 'radii' containing all bonds between included atoms and are constructed as detailed in the "Methods", unless otherwise stated. The 'radius' ( $r$ ) of an atom environment is the maximum allowed topological distance between the center atom and any atom in the original structure that is part of the atom environment, and is measured as the number of bonds along the shortest path [55]. The analysis of PubChem Compound and Substance was performed with respect to atom environments from topological radii zero (i.e., atom type) up to three. As a means of comparison, atom environments of $r=3$ are essentially equivalent to those generated by the popular ECFP_6 type extended connectivity fingerprints [34] or Morgan Fingerprints of $r=3$ [56]. Atoms are characterized by atomic number, formal charge, implicit hydrogen atom count, explicit degree (number of explicitly connected atoms), and their valence (the sum of implicit hydrogen count and bond orders of incident bonds). Bonds are distinguished as single, double or triple covalent bonds. Both atoms and bonds were further characterized by their participation in aromatic systems. To ensure that these properties are set consistently, pre-processing is performed that converts all explicit 
hydrogen atoms to implicit hydrogen atom counts (ignoring isotopes and annotated stereo chemistry) and that perceives aromaticity. Atom environment frequencies are specified by incidence, being the absolute number or relative percentage of the 104,669,789 substance or 46,704,121 compound records considered in this study where a molecular fragment is present.

\section{Known Chemistry ('then and now') Comparison}

To contrast the current state of known chemistry ('now') with that from a little more than 40 years ago ('then'), we generated atom environments of radius $r=1$ for all structures in PubChem Compound. To achieve a direct comparison, we used the same atom and bond types as Adamson et al. [47] when analyzing the Crowe et al. data [46]. In these earlier fragmentation studies: atoms were distinguished by atomic number; and bonds were classified as single/double/triple and as chain/ring bonds, respectively, and by an aromatic-ring bond type. For better discrimination between this then to now comparison from other results of our study, this particular size of atom environment generated with these particular atom types and bond types will be referred to as 'augmented atoms', as in the 1971 study. Based on this classification scheme, the 1971 study found a total of 2,331 unique augmented atoms in a collection of 28,799 molecules randomly sampled from the CASRS. To ensure comparability, we applied the same pre-filtering steps to structures as performed in the original study: entries with more than 100 atoms were omitted, and only structures containing atoms between 1 and 4 explicit connections were allowed, yielding 46,605,207 allowed and 98,914 rejected compound records from the $46,704,121$ compound records in PubChem. In addition, terminal atoms were allowed as center atoms of atom environments. Aromaticity was perceived using the OEChem $\mathrm{C}++$ toolkit [57] aromaticity model OEAroModelMDL, which allows only six-membered rings of carbon and nitrogen to be aromatic, provided they satisfy the 'Hückel $4 \mathrm{n}+2^{\prime}$ rule $[58,59]$ (i.e., atoms are $\mathrm{sp}^{2}$-hybridized). Even though no aromaticity definition was supplied in the 1971 study, due to its simplicity, it is our opinion that this model might be closest to the perception of aromaticity at that time [60].

Using this earlier analysis method, 18,381 unique $r=1$ augmented atoms were identified in PubChem Compound. (Please note that not adhering to the original study's prefiltering steps has virtually no effect on the results with respect to incidences and occurrences; however, the number of augmented atoms increases slightly to 18,503.) The most frequent augmented atom is part of an aromatic system and contains three aromatic carbon atoms and an implicit hydrogen atom (represented as 'c:c:c' in SMILES). Compared to an incidence of $61.2 \%$ and an occurrence of
$18.0 \%$ in 1971 , today it is present in $83.7 \%$ of all structures and accounts for $20.3 \%$ of all fragments. This indicates that today a higher fraction of known structures contain aromatic systems. Further evidence can be found by looking at the augmented atom ' $\mathrm{c}: \mathrm{c}(-\mathrm{C}): \mathrm{c}$ ', a 'branching' fragment from an aromatic system, ranked sixth most frequent in 1971 is now third most frequent today. Its incidence grew from 38.4 to $66.7 \%$, meaning that this augmented atom is present in almost twice as many structures today. In accordance with these findings, its occurrence increased from 2.9 to 4.6\%. Unfortunately, the study by Adamson et al. from 1971 does not contain a complete list of identified augmented atoms or the list of original structures. Consequently, we were unable to duplicate their study and perform a detailed comparison of the results to identify augmented atoms unique to each of the sets. Such a comparison could possibly identify changes in utilized and preferred chemistry with greater specificity. However, the juxtaposition of frequencies of elements encountered in both repositories (for the 1971 analysis, data published by Crowe et al. [46] is used) as presented in Table 1 reveals similarities and differences at the level of element distribution.

Comparing then (1970 Crowe et al. study [46]) and now (2013 PubChem Compound), carbon is unchanged. It is the most abundant element found in chemical structures, accounting for $74 \%$ of all atoms and found in more than $99 \%$ of all structures. The story is different for oxygen. It accounts for a decreased percentage of all atoms (13.5\% then and $11.3 \%$ now) but, interestingly, the fraction of structures containing oxygen have increased $(82.6 \%$ then and $91.5 \%$ now). The change is even more dramatic for nitrogen. It accounts for a substantially larger percentage of all atoms $(7.3 \%$ then and $10.2 \%$ now $)$ and is present in substantially more chemical structures $(64.2 \%$ then and 91.5\% now). Combined, these three elements ( $\mathrm{C}, \mathrm{N}, \mathrm{O})$ account for nearly all atoms both then (94.8\%) and now (95.7\%). Other noteworthy changes include the increased presence in structures the elements sulfur $(19.9 \%$ then and $34.2 \%$ now) and fluorine (10.0\% then and $18.1 \%$ now). The reported incidence of other elements was limited in the 1971 study, preventing a more complete analysis.

Comparison of the top-10 augmented atoms between then (Fig. 1) and now (Fig. 3) is very interesting. They show oxygen double bonded to carbon $\left({ }^{\circ} \mathrm{O}=\mathrm{C}^{\prime}\right)$ jumping from a ranking of 5 th to 2 nd. Three new now top-10 augmented atoms (in terms of incidence) appear, all containing nitrogen, including secondary amine $($ ' $\mathrm{C}-\mathrm{N}-\mathrm{C}$ ', ranked 5th), amide (' $\mathrm{C}-\mathrm{C}(=\mathrm{O})-\mathrm{N}$ ', ranked 6 th), and aliphatic amine (' $\mathrm{C}-\mathrm{C}-\mathrm{N}$ ', ranked 7 th). These replace the then augmented atom of aliphatic carbon chain (' $\mathrm{C}-\mathrm{C}-\mathrm{C}$ ', ranked 4th), aromatic amine ('c:c(-N):c', ranked 9th), and aliphatic carbonyl (' $\mathrm{C}-\mathrm{C}(=\mathrm{O})-\mathrm{C}$ ', ranked 10th). In addition, between then and now the 6th ranked fragment 
Table 1 Elemental analysis and comparison

\begin{tabular}{|c|c|c|c|c|c|}
\hline \multicolumn{3}{|l|}{ CASRS (1970) } & \multicolumn{3}{|c|}{ PubChem compound (2013) } \\
\hline Atomic symbol & Occurrence (\%) & Incidence (\%) & Atomic symbol & Occurrence (\%) & Incidence (\%) \\
\hline C & 74.006 & 99.644 & C & 74.129 & 99.922 \\
\hline O & 13.519 & 82.578 & N & 10.238 & 91.629 \\
\hline N & 7.258 & 64.165 & $\mathrm{O}$ & 11.288 & 91.525 \\
\hline $\mathrm{F}$ & 1.690 & 10.020 & $\mathrm{~S}$ & 1.564 & 34.171 \\
\hline$S$ & 1.302 & 19.925 & $\mathrm{Cl}$ & 0.933 & 19.184 \\
\hline $\mathrm{Cl}$ & 1.208 & 14.032 & $\mathrm{~F}$ & 1.350 & 18.153 \\
\hline$P$ & 0.282 & n.s. & $\mathrm{Br}$ & 0.266 & 6.468 \\
\hline $\mathrm{Br}$ & 0.262 & n.s. & $P$ & 0.060 & 1.159 \\
\hline $\mathrm{Si}$ & 0.114 & n.s. & $\mathrm{Si}$ & 0.056 & 1.058 \\
\hline । & 0.077 & n.s. & । & 0.041 & 0.968 \\
\hline B & 0.063 & n.s. & B & 0.017 & 0.335 \\
\hline Sn & 0.026 & n.s. & $\mathrm{Na}$ & 0.012 & 0.211 \\
\hline $\mathrm{Se}$ & 0.020 & n.s. & $Y$ & 0.004 & 0.071 \\
\hline As & 0.020 & n.s. & Sn & 0.003 & 0.064 \\
\hline
\end{tabular}

Incidence is used for ranking. Occurrence is calculated based on counts of non-hydrogen atoms. Incidence refers to the number of structures in the respective repository that contained at least one atom of the particular element. Frequencies determined in PubChem Compound are compared to a 1970 analysis of the Chemical Abstracts Service Registry System (CASRS) by Crowe et al. [46]. Data not supplied by Crowe et al. is indicated as 'not specified' (n.s.). Even though the CASRS data was generated using only 28,963 of 596,367 available compounds, the authors found their data to be nearly identical with that obtained by others from the full set.

\begin{tabular}{|c|c|c|c|c|c|}
\hline & & $\begin{array}{l}O \\
\mathbb{C} \\
\end{array}$ & & $\begin{array}{l}\text { C } \\
\text { I } \\
\text { C }\end{array}$ & \\
\hline Occurrence & $20.3 \%$ & $5.0 \%$ & $4.6 \%$ & $5.4 \%$ & $2.4 \%$ \\
\hline Incidence & $83.7 \%$ & $73.9 \%$ & $66.7 \%$ & $65.7 \%$ & $46.2 \%$ \\
\hline & & & $\begin{array}{l}\text { O } \\
\text { I } \\
\text { C }\end{array}$ & & Ring \\
\hline Occurrence & $2.4 \%$ & $2.4 \%$ & $2.2 \%$ & $2.3 \%$ & 3.2 \\
\hline Incidence & $45.2 \%$ & $44.1 \%$ & $42.1 \%$ & $42.1 \%$ & \\
\hline
\end{tabular}

Fig. 3 Top-10 most frequent 'augmented atoms' in PubChem Compound. PubChem Compound records were fragmented with atom type and bond type definitions identical to those used by Adamson et al. [47]. Incidence was used for ranking, calculated based on 46,605,207 compound records. Dashed lines indicate aromatic bonds as perceived using the aromaticity model OEAroModelMDL in the OpenEye Scientific Software, Inc. OEChem C++ toolkit [57].

bumps up to 3rd and the 2nd, 7th, 8th, and 3rd ranked fragments then decreased to become the 4th, 8th, 9th, and 10th ranked fragments now, respectively.

Beyond the top-10, this comparison shows that since 1970 the number of unique augmented atoms generated has increased by a factor of 7.9. (As stated later, this factor of 7.9 should be considered an upper bound estimation.) This increase in fragment diversity shows that, in the last 40 years, chemists have increased their ability considerably to generate unique combinations of elements and their binding patterns. In addition, and as reflected in the top-10, chemists have become much more adept at working with organic chemicals containing oxygen and nitrogen bonds to the point that many chemicals now contain these. (See also part of the "Atom environment rate of growth" below for discussion on over and under estimation of atom environments in this analysis.) For completeness, the full data of the elemental analysis of Compound is provided as supporting information in Additional file 1: Figure S1 and Table S1. The full list of 
augmented atoms used in this then and now comparison with their respective incidence is provided as supporting information in Additional file 2.

\section{Analysis of PubChem Substance and Compound}

Analysis of PubChem chemical substance descriptions (PubChem Substance: 104,669,789 records) and the unique set of chemical structures after PubChem normalization processing (PubChem Compound: 46,704,121 records) was performed. These two collections were examined according to the data preprocessing and analysis approach as described in the "Methods". Atoms were characterized by atomic number, formal charge, implicit hydrogen count, explicit degree (number of neighbors), valence (bond order sum including implicit hydrogen atom counts), and aromaticity. Bonds were differentiated as single, double, triple, or aromatic bonds. Atom environments were generated for radii $r=0$ (atom types), $r=1, r=2$, and $r=3$, where the topological radius $(r)$ is the maximum allowed topological distance between the center atom and any atom in the original structure that is part of the atom environment, as measured by the number of bonds along the shortest path [55].

PubChem Substance contained 8,135 $(r=0), 299,609$ $(r=1), 5,453,889(r=2)$, and $26,988,962(r=3)$ unique atom environments, respectively. PubChem Compound contained 1,583 $(r=0), 109,306(r=1), 4,559,587$ $(r=2)$, and 25,115,177 $(r=3)$ unique atom environments, respectively. In Fig. 4, the growth of the number of unique atom types and atom environments in Substance and Compound with increasing substance identifier (SID) and compound identifier (CID) are shown. One cannot read too much into Fig. 4, as the plot of increasing identifier indicates only a historic growth of atom environments with respect to when new/unique records were added to PubChem. For example, new contributors may give "new" structures to PubChem that were known already for some time. When examining Fig. 4, it may seem counterintuitive that for radii $r=2$ (Fig. 4c) and $r=3$ (Fig. 4d) a particular fraction of Compound has more unique atom environments than Substance; however, there is no direct correlation

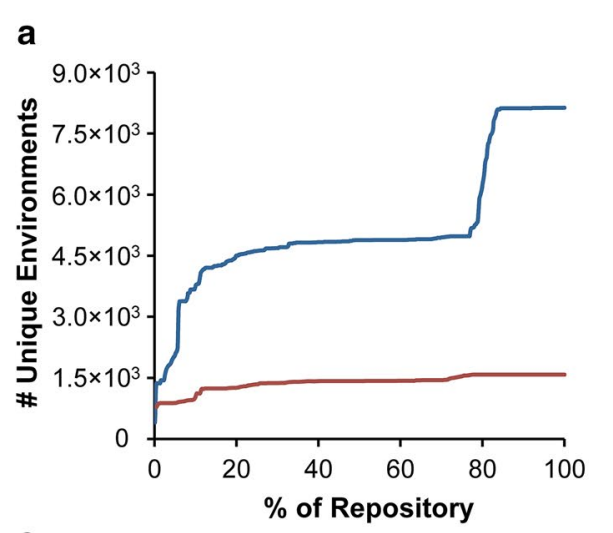

\section{b}
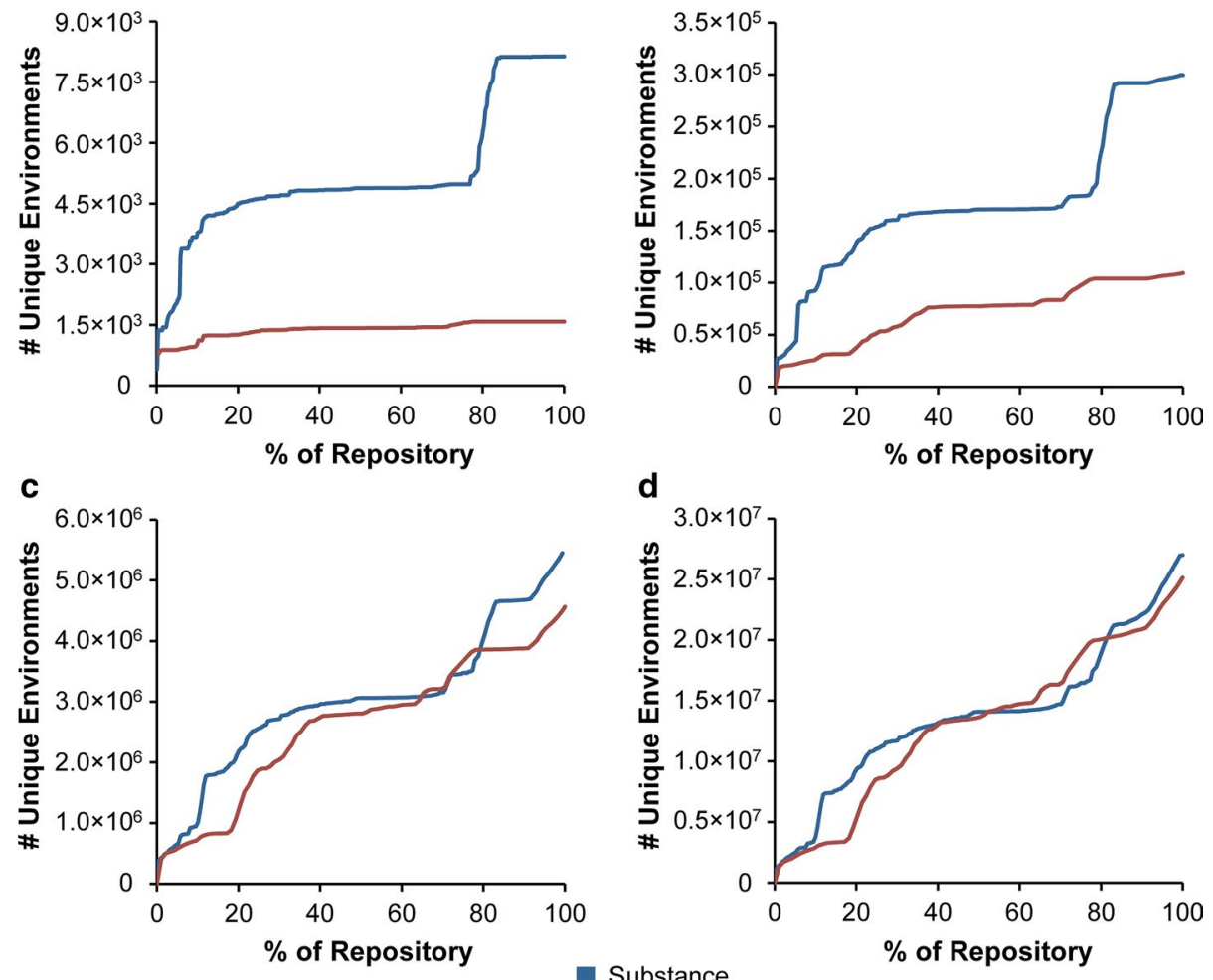

d

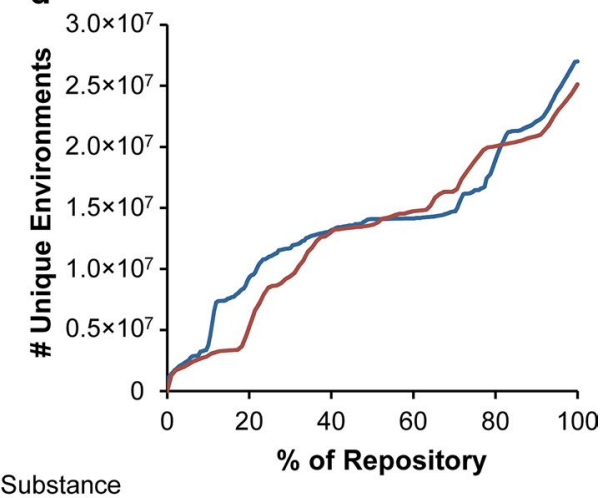

Substance

Compound

Fig. 4 Atom environment statistics. Number of unique atom environments with radius: $\mathbf{a} r=0$ (atom types), $\mathbf{b} r=1 ; \mathbf{c} r=2 ;$ and $\mathbf{d} r=3$. Values are per fraction of the respective database, PubChem Substance (104,669,789 SIDs), and PubChem Compound (46,704,121 CIDs). Total fragment counts for $r=0,1,2,3$ are: 8,135 atom types, 299,609, 5,453,889, and 26,988,962 atom environments, respectively, for Substance; and 1,583 atom types, $109,306,4,559,587$, and 25,115,177 atom environments, respectively, for Compound. 
between the percentage of Substance and Compound, per se, due to the duplicity and order of chemical structures in Substance. Compound is the unique content of Substance (after PubChem normalization processing) and Substance contains more than twice the number of records as Compound (104 million SIDs vs. 46 million CIDs, at the time of this study). Therefore, there should be no assumption that there is a direct correlation between the two database percentages.

\section{Atom type ( $r=0$ atom environment) statistics}

PubChem Substance and Compound contain atom types $(r=0)$ of all elements from atomic number 1 (hydrogen) to 109 (meitnerium). In total, 8,135 different atom configurations occur in Substance, with 2,644 (32.5\%) being singletons (i.e., occurring in only one substance record). These respective numbers are significantly lower for Compound, which contains 1,583 different atom types, $167(10.6 \%)$ of which are singletons. Rank/frequency plots for atom types in Substance and Compound are shown in Fig. 5. Their dispersal among elements is found in Fig. 6. In total, the largest number of different atom types in the Substance database is based on carbon $(820$ atom types, $10.1 \%$ ), while in the Compound database, the largest number is based on phosphorous (63 atom types, $4.0 \%)$.

The top-10 most frequent atom types $(r=0)$ in Substance and Compound are shown in Fig. 7. Similar to the earlier 'then and now' comparison, all of these top-10 atom types are based on carbon, nitrogen or oxygen. In addition, these atom types are identical between Substance and Compound databases but ranked in a slightly different order, in part, due to the duplicity of the chemical structures in Substance records. Figure 7 also shows that a very small minority of atom types (eight in Substance and nine in Compound) occur in more than 50\% of all records in the respective database. The two most frequent atom types contain carbon in an aromatic system, one being additionally bonded to a non-hydrogen atom (Substance 92.1\%; Compound 90.6\%) and the other to a hydrogen atom (Substance: 90.7\%; Compound $89.2 \%$ ). If aromaticity is ignored, the Substance atom types (i) and (vi) and Compound atom types (i) and (vi) would be identical.

The 8,135 atom types reflect the heterogeneity of chemical representations in Substance but also the

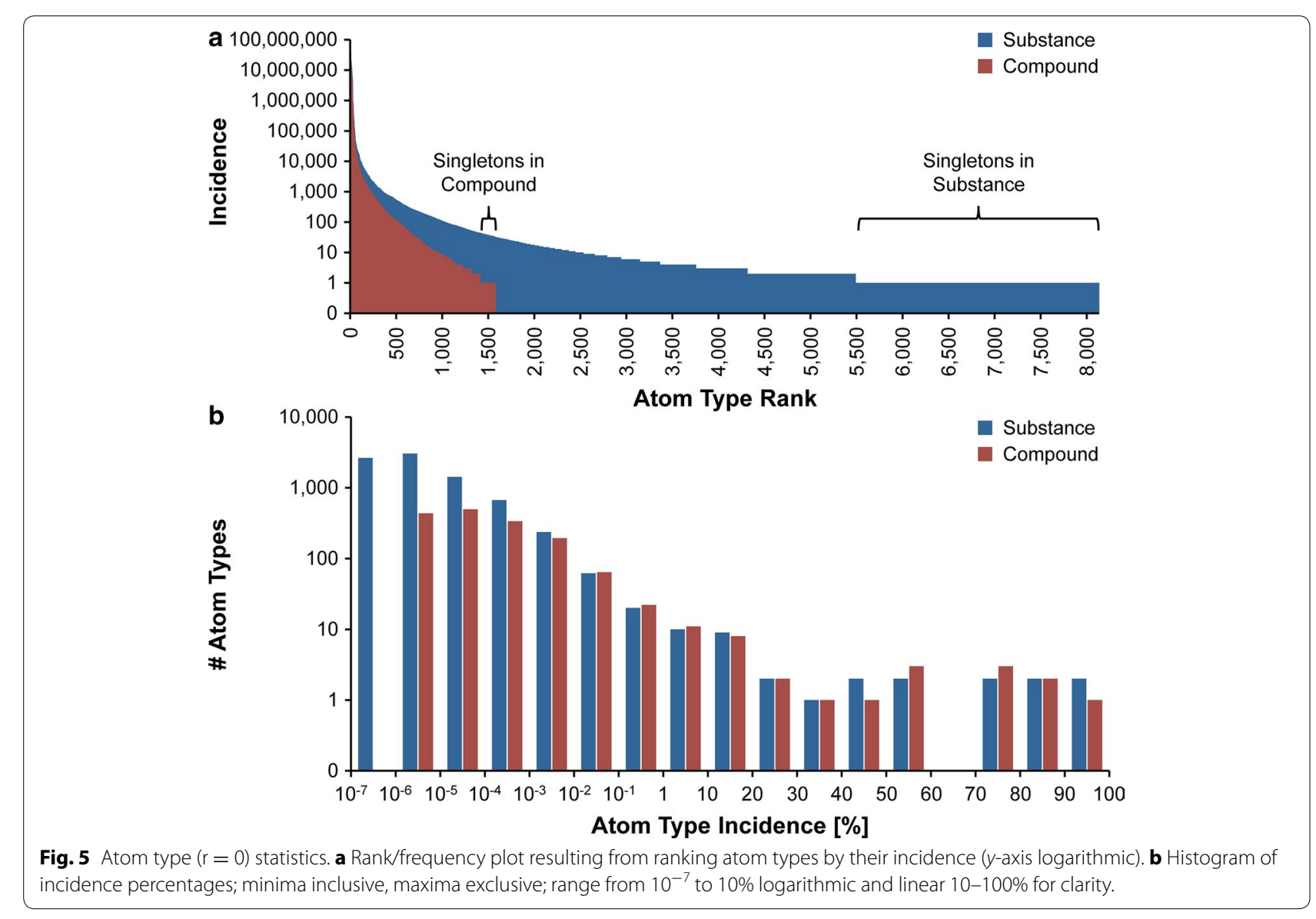




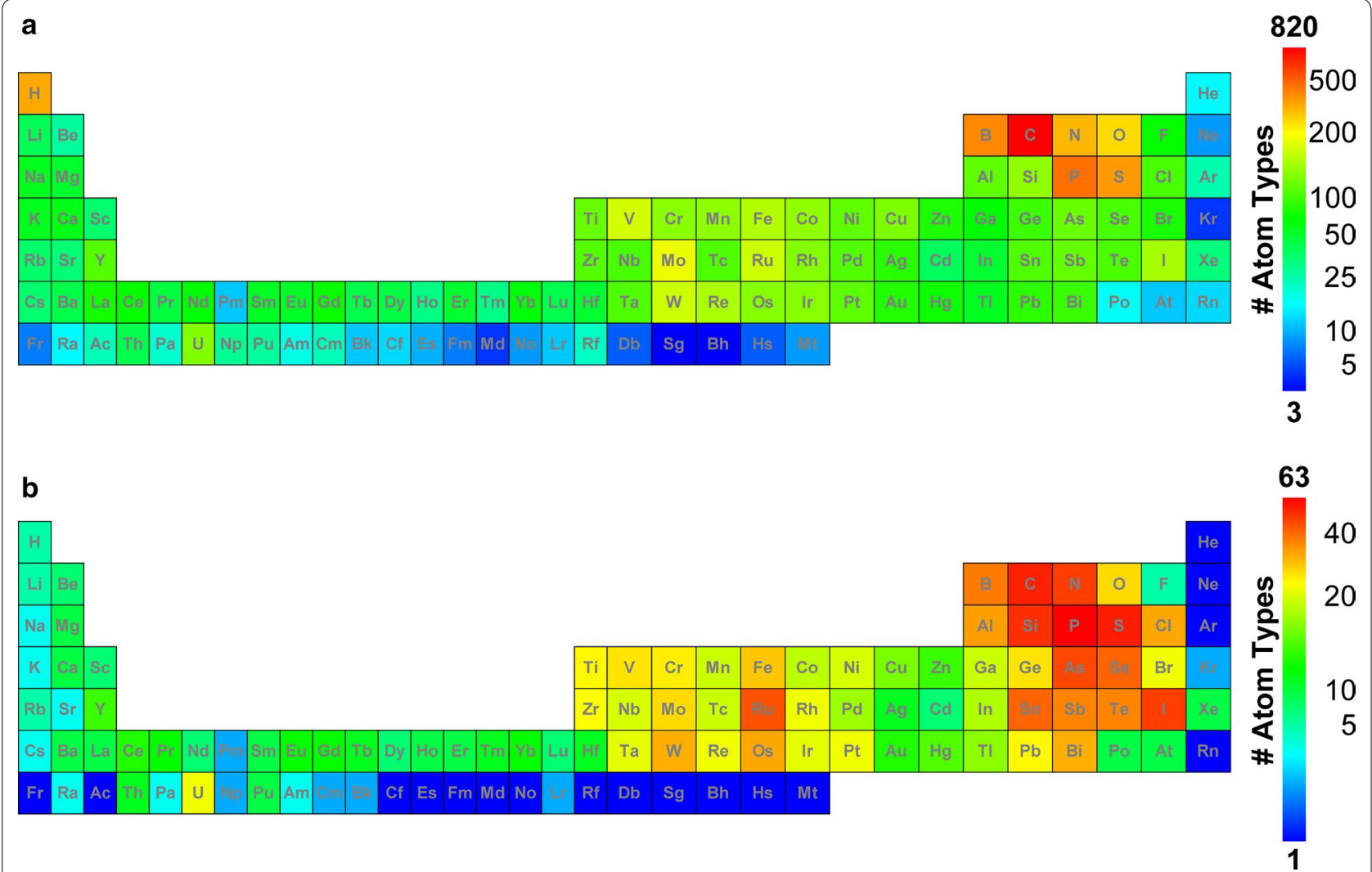

Fig. 6 Atom type $(r=0)$ counts per element. Number of atom types per element. a Substance; b Compound. Color coding normalized to the respective highest/lowest atom type count for clarity. Logarithmic-based color scale is used for better differentiation between low counts.

bizarre. Hydrogen is present in no less than 324 configurations of which 166 (51.2\%) are singletons; exemplary atom types unique to Substance are shown in Fig. 8a. PubChem standardization protocols reject 320 of the 324 encountered hydrogen configurations. As such, only four different hydrogen-only atom types exist in Compound: monohydrogen radical, elemental hydrogen in $\mathrm{H}_{2}$, hydride ion $\left(\mathrm{H}^{-}\right)$, and hydron ion $\left(\mathrm{H}^{+}\right)$. It may be noteworthy that these hydrogen species are present in the Compound database as explicit atoms despite (explicit to implicit) hydrogen suppression procedures.

In the case of halogen atom types found in Substance, there are 51 for fluorine [16 (31.4\%) singletons], 74 for chlorine [16 (21.6\%) singletons], 123 for bromine [56 (45.5\%) singletons] and 57 for iodine [17 (29.8\%) singletons]. Exemplary atom types unique to Substance are shown in Fig. 8b. In Compound, the number of different halogen atom types is substantially lower, with 4 fluorine atom types [0 singletons], 32 chlorine atom types [7 (21.9\%) singletons], 49 bromine atom types [12 $(24.5 \%)$ singletons] and 21 iodine atom types [5 (23.8\%) singletons].
Even though noble gases initially were thought to be chemically inert, today, a few noble gas compounds are known [61-67]. Nonetheless, the 206 noble gas atom types identified in Substance seems irrationally high. Exemplars of atom types per noble gas unique to Substance are shown in Fig. 8c.

The 2,644 singleton atom types in Substance can highlight peculiar configurations. For instance, in the case of carbon, there are 351 singletons $(13.3 \%$ of all singleton cases), with formal charges ranging from -99 to +291 and valences ranging from 0 to 254 (examples shown in Fig. 8d). For other elements, the situation is similar, as illustrated for organic elements $(\mathrm{H}, \mathrm{C}, \mathrm{N}, \mathrm{O}, \mathrm{F}, \mathrm{P}, \mathrm{S}, \mathrm{Cl}, \mathrm{Br}$, or I) in Table 2. In total, only 71 of 2,644 (2.7\%) singleton atom types pass the PubChem "allowed" valence list.

The large number of strange atom types specific to Substance sparked an interest in their origin. Unusual connectivity patterns (and, therefore, valences) are commonly found in depictions between metals and organic elements, where non-covalent interactions are represented using covalent bonds in accordance with the structure representation rules of a given organization. If one ignores such chemistry and considers only the atom 
a

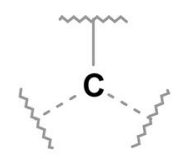

i) $92.12 \%$<smiles>CC=C(C)C(C)C</smiles>

vi) $75.67 \%$

b

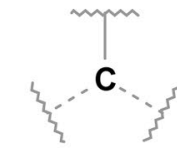

i) $90.63 \%$

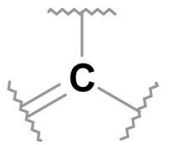

vi) $74.73 \%$<smiles>CCCCC(C)C</smiles>

ii) $90.73 \%$

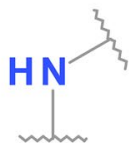

vii) $54.86 \%$<smiles>CCCC(C)CC</smiles>

ii) $89.15 \%$

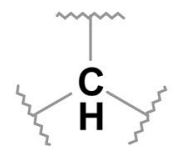

vii) $57.85 \%$<smiles>CCCC(C)C</smiles>

iii) $83.88 \%$

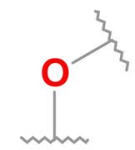

viii) $51.79 \%$<smiles>O=C1CCCCC1</smiles>

iv) $81.02 \%$<smiles>CCC(CC)C(CC)CC</smiles>

ix) $48.52 \%$<smiles>CCCCC(C)C</smiles>

iii) $87.41 \%$

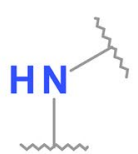

viii) $56.14 \%$<smiles>CC1CC1</smiles>

iv) $79.92 \%$

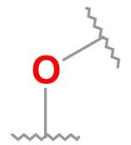

ix) $51.11 \%$<smiles>CC1CC1</smiles>

v) $77.50 \%$<smiles>CCN(CC)CC</smiles>

x) $40.32 \%$

Fig. 7 Top-10 most frequent atom types $(r=0)$ in PubChem. Percentages indicate incidence of the atom type in the respective database: a Substance; and $\mathbf{b}$ Compound. Light gray bonds for clarification of connectivity and valence. Dashed lines indicate aromatic bonds as perceived using the aromaticity model OEAroModelOpenEye in the OpenEye Scientific Software, Inc. OEChem C++ toolkit [57].

types of organic atoms with bonds only to other organic atoms, the total count of 8,135 Substance atom types collapses down to 2,727 (33.5\%). Of these 2,727 Substance "organic-only" atom types, 2,422 (88.8\%) are considered "invalid" by PubChem and 1,144 (47.2 \%) of these "invalid organic-only" atom types occur in only a single substance (i.e., are exceedingly rare). In total, 534,968 substances from 119 depositors have one or more of these "invalid organic-only" atom types. The top-10 contributors of these are shown in Fig. 9. Please note that the presence of these "invalid organic-only" atom types does not necessarily indicate a lapse of quality of these resources. It may, in some cases, suggest that PubChem prefers a different chemistry representation than that provided, as "invalid organic-only" includes atom types such as the pervasive pentavalent nitrogen (e.g., as found in the "*: $\mathrm{N}(=\mathrm{O})=\mathrm{O}$ " nitro group representation) commonly found in chemical databases.

Nearly $75 \%$ of the +500 thousand substances with "invalid organic-only" atom types are from three PubChem contributors: SCRIPDB [179,784 (33.6\%)] [68, 69], IBM [131,014 (24.5\%)] [70], and ChemSpider [85,894 (16.1\%)] [71]. Examples from SCRIPDB are depicted in Figs. 10a (i-x), 8a (ii-iii), b (iv), c (i-iv), (vi), and d (i-iv). Examples from IBM can be seen in Figs. 10b $(i-x)$ and 8a (i). Examples from ChemSpider are found in Figs. 10c $(\mathrm{i}-\mathrm{x})$ and $8 \mathrm{~b}$ (i). For SCRIPDB, IBM and ChemSpider, the relative incidence of the ten most frequent invalid organic-only atom types are presented in Fig. 11 relative to the respective $(\mathrm{i}-\mathrm{x})$ atom types depicted in Fig. 10.

In total, 2,181 different "organic-only" atom types encountered in substances deposited by SCRIPDB do not pass the PubChem valence list. SCRIPDB provides chemical structures found in the complex work units (e.g., Figures) of USPTO (United States Patent Office) patent documents. The most frequent one, found in $21.7 \%$ of the 179,784 substances with "invalid organic-only" atom types (referred to henceforth as "invalid substances"), is uncharged, tetra-coordinated, tetra-valent nitrogen that gets an added implicit hydrogen atom during pre-processing. This configuration is not allowed in the PubChem valence list, but is salvageable by standardization protocols with a simple fix: the implicit hydrogen is removed during structure standardization, and the nitrogen atom gets assigned a positive charge. The next most frequent atom types are tri-coordinated and tri-valent oxygen (16.9\% of invalid substances), tri-coordinated and penta-valent carbon (8.8\% of invalid substances), and tetra-coordinated and penta-valent carbon $(8.1 \%$ of invalid substances). These atom types lead to the rejection 


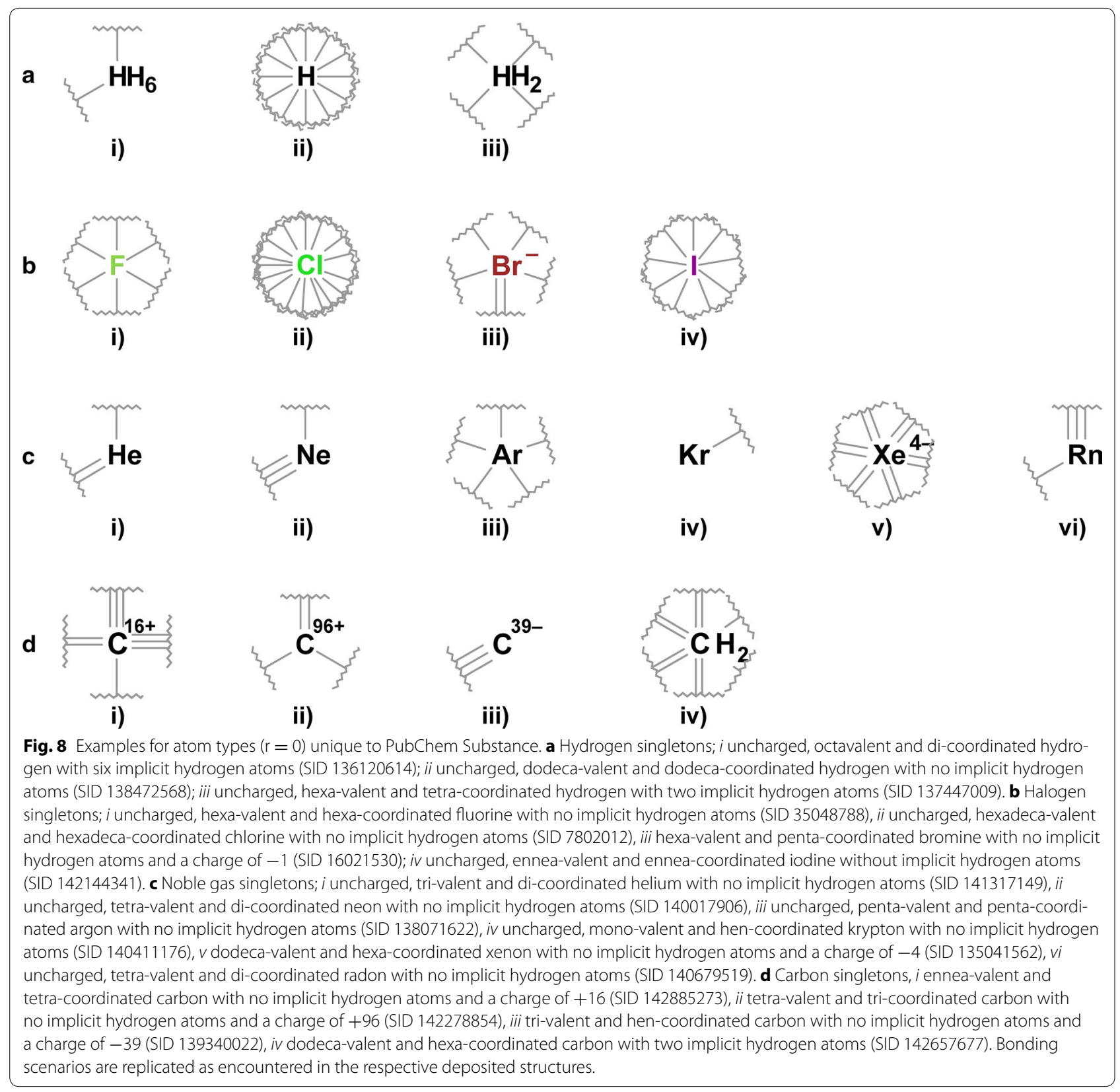

of a substance during standardization. The case of tetracoordinated, tetra-valent and uncharged nitrogen $(6.8 \%$ of invalid substances) is analogous to the most frequent atom type. Di-coordinated and di-valent hydrogen cases (4.7\% of invalid substances) are not salvageable by structure standardization. The same goes for di-coordinated and tri-valent oxygen (3.9\% of invalid substances) and di-coordinated and di-valent fluorine $(3.7 \%$ of invalid substances). Penta-coordinated and penta-valent carbon (3.5\% of invalid substances) causes the respective substances also to be rejected. Tri-coordinated, tetra-valent carbon with a positive charge (3.4\% of invalid substances) does not exist in the PubChem valence list and is not salvaged by standardization. A total of 1,138 of the 2,181 (52.2\%) rejected atom type cases identified in substances provided by SCRIPDB occur in only a single substance record.

There are 116 "invalid organic-only" atom types found in 131,014 substances provided by IBM. The chemical structures from IBM are pulled from patent and biomedical literature documents. Contrary to SCRIPDB, the number of affected substances is dominated by a 
Table 2 Properties of organic atom type singletons

\begin{tabular}{|c|c|c|c|c|c|c|}
\hline \multirow[t]{2}{*}{ Atomic number } & \multirow[t]{2}{*}{ Atomic symbol } & \multirow[t]{2}{*}{ Number singletons } & \multicolumn{2}{|l|}{ Charge } & \multicolumn{2}{|l|}{ Valence } \\
\hline & & & Minimum & Maximum & Minimum & Maximum \\
\hline 1 & $\mathrm{H}$ & 166 & -99 & 126 & 0 & 91 \\
\hline 6 & C & 351 & -99 & 291 & 0 & 254 \\
\hline 7 & $\mathrm{~N}$ & 111 & -67 & 7 & 0 & 36 \\
\hline 8 & $\mathrm{O}$ & 82 & -94 & 123 & 0 & 50 \\
\hline 9 & $\mathrm{~F}$ & 16 & -2 & 0 & 1 & 137 \\
\hline 15 & P & 231 & -99 & 55 & 0 & 233 \\
\hline 16 & $S$ & 169 & -99 & 127 & 0 & 234 \\
\hline 17 & $\mathrm{Cl}$ & 16 & -2 & 5 & 0 & 64 \\
\hline 35 & $\mathrm{Br}$ & 17 & -3 & 1 & 0 & 8 \\
\hline 53 & । & 56 & -5 & 7 & 0 & 171 \\
\hline
\end{tabular}

Organic elements are used as examples.

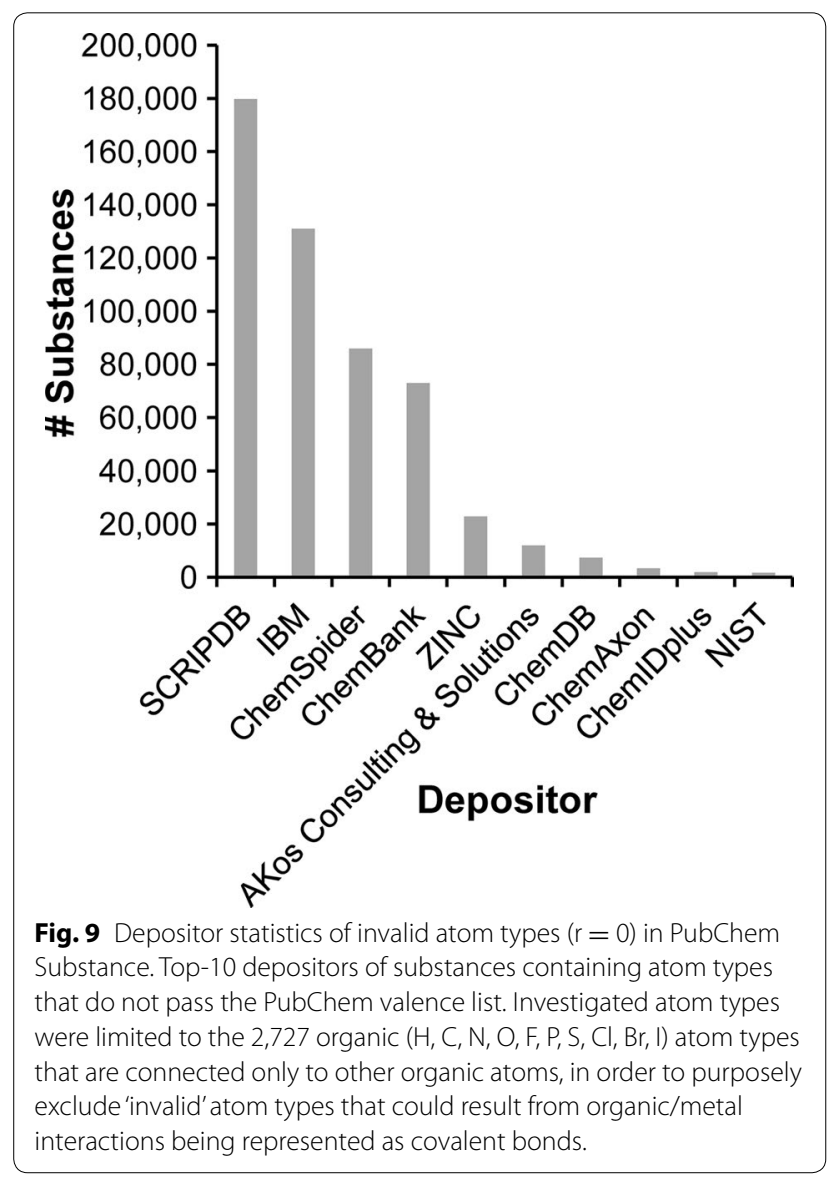

single atom type: uncharged, tri-coordinated and pentavalent nitrogen, which is present in $79.1 \%$ of the affected substances. Inspection of the substances provided to PubChem revealed that this is due to the configuration of nitro groups as "* $\mathrm{N}(=\mathrm{O})(=\mathrm{O})$ ", a common representation approach but deemed invalid by PubChem which favors the "* $[\mathrm{N}+](=\mathrm{O})-[\mathrm{O}-]$ " representation. This is remedied by PubChem standardization, where the configuration of the nitro group with penta-valent nitrogen is modified to be the PubChem-preferred representation. Five of the top-10 ranked "invalid organic-only" atom types are identical with those identified for SCRIPDB in Fig. 10. Substances containing tetra-coordinated and tetravalent oxygen $(0.5 \%$ of 131,014 substances $)$ get rejected by standardization. Tetra-coordinated and penta-valent nitrogen is analogous to the most frequent atom type for IBM that is rejected by the PubChem valence list. During structure standardization, this can be resolved if the respective atom is double-bonded to an oxygen atom by modifying this atom type to a charge-separated representation of the double bond to form a tetra-coordinated and tetra-valent nitrogen that carries a positive charge. Tri-coordinated and tetra-valent oxygen $(0.3 \%$ of 131,014 substances) is rejected by the PubChem valence list. Dicoordinated and tetra-valent nitrogen gets assigned an implicit hydrogen count of +1 during pre-processing $(0.2 \%$ of 131,014 substances) that later is replaced by a positive charge during standardization. In total, 40 of the 116 (34.5\%) invalid atom types identified in substances deposited by IBM occur in only a single substance.

The 85,894 substances with "invalid organic-only" atom types provided by ChemSpider contain at least one of 282 offending atom types. The most frequent one, tri-coordinated tetra-valent nitrogen that is uncharged and has no implicit hydrogen atoms (50.1\% of 85894 substances), is an annotated nitrogen radical. It occurs 8,425 times in the context of nitro groups being represented as "*: $[\mathrm{N}]$ $(=\mathrm{O})\left(-\left[\mathrm{O}^{-}\right]\right)$, and 100 times in other contexts. This representation was previously not handled by the PubChem structure standardization protocols, and consequently, 


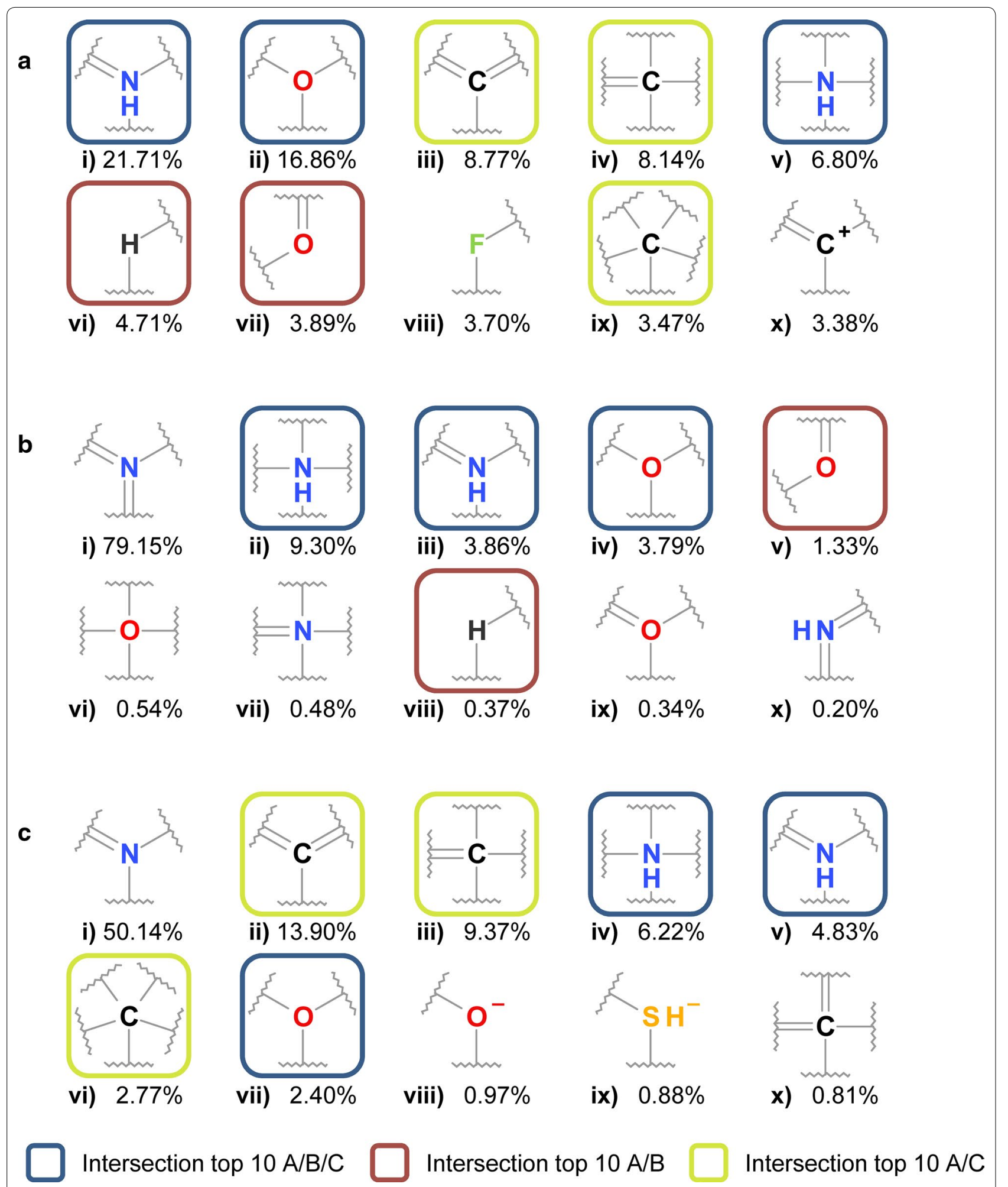

Fig. 10 Top-10 most frequent invalid atom types $(r=0)$ from select data sources. Shown are the top-10 most frequent atom types that fail the PubChem valence list for the top-3 depositors of substances containing failing atom types: a SCRIPDB, $\mathbf{b}$ IBM, and $\mathbf{c}$ ChemSpider. Atom types are ranked by their relative frequency in substances deposited by the respective contributor that have at least one atom that does not pass the valence list. Atom type incidences were calculated for each depositor separately. 


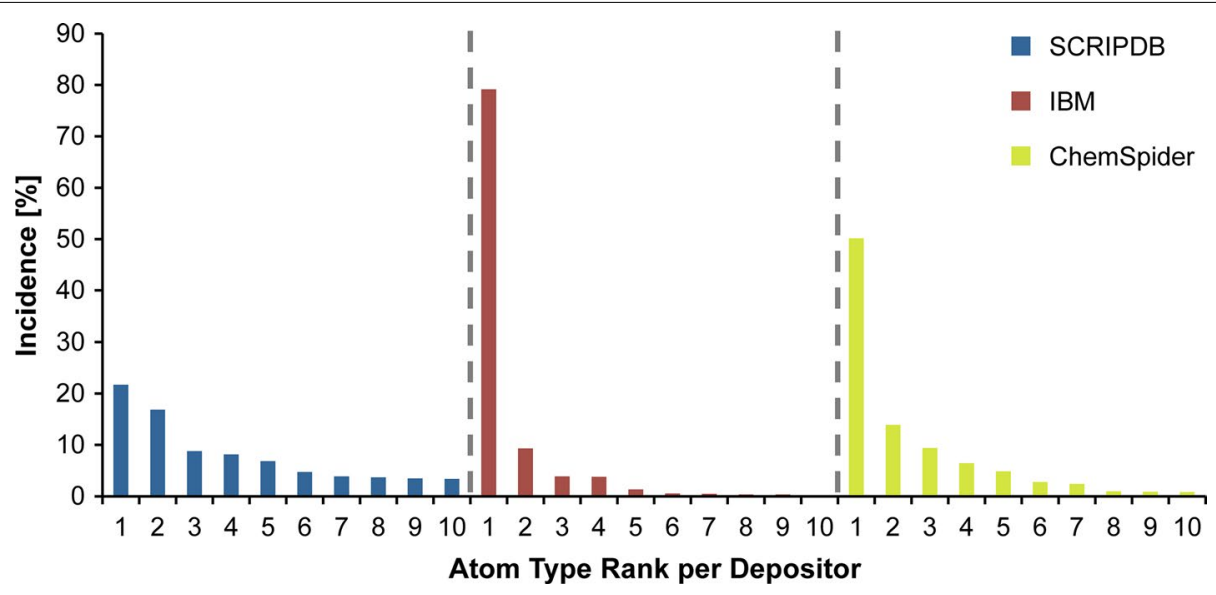

Fig. 11 Statistics of invalid atom types $(r=0)$ from select data sources. For the top-3 depositors of substances containing invalid 'organic-only' atom types, the incidence of the respective top-10 most frequent invalid atom types is shown. Incidence is calculated for each contributor separately based on the total number of substances containing such atom types. For the corresponding atom types, see Fig. 10.

affected substances were rejected. The top-10 ranked invalid atom types identified in substances deposited by ChemSpider share three types with the top-10 ranked atom types from SCRIPDB and IBM, and three other atom types with the top-10 ranked atom types just from IBM. As a complementary invalid atom type, di-coordinated and di-valent oxygen carrying a negative charge occurs in $0.97 \%$ of 85,894 substances. These respective cases cannot be salvaged, as it is not clear whether connectivity information or formal charge should have precedence such that this atom type can be modified in order to pass the PubChem valence list. The hydrogen atom in tri-coordinated and tri-valent negatively charged sulfur is actually added during pre-processing, it is deposited as di-coordinated, di-valent and carrying a negative charge. Both configurations are neither in the PubChem valence list nor treated by the standardization protocols and are rejected during standardization. A consequence of the selection criteria for investigated atom types is that all bonds in tetra-coordinated hexa-valent carbon $(0.81 \%$ of 85,894 substances) are considered to be covalent and this atom type does not pass the valence check during structure standardization. Of the invalid atom types encountered in substances deposited by ChemSpider, 81 of 282 (28.7\%) invalid atom types occur in only one substance.

These top-10 cases of "invalid organic-only" atom types help to highlight several things. Firstly, Fig. 10 suggests that simple examination of atom types in a given molecule collection can be helpful to identify molecules that may be considered invalid as depicted. Secondly, as indicated by highlighted overlap in Fig. 10, it demonstrates that different organizations can share or differ in preference for particular "invalid" molecule representations, with each organization potentially providing previous unimagined atom configurations that may or may not be salvageable. Thirdly, some of these atom environments that are technically "invalid" from the PubChem perspective can be readily fixed/normalized to the PubChem preferred atom environment. For example, if one removes an implicit hydrogen atom and adds a positive charge, it would make the nitrogen atom in the Fig. 10a (i, v), b (ii, iii, $x$ ), and c (iv, v) cases "valid". Indeed, PubChem standardization protocols can "correct" the nitrogen atom type as highlighted in these cases, however, it is worth mentioning that such fixes consider the larger atom environment $(r>0)$ and often modify several atom types and respective bonding patterns between them, as opposed to a systemic fix considering only a single atom type $(r=0)$. (Fixes at the $r=0$ atom environment level should be considered to be ill advised as they may complicate or prevent correction of a larger functional group representation.) Lastly, as suggested in Fig. 11, differences in opinion about preferred representation may affect a large number of structures, but the majority of those differ in only a small number of atom types (in this case, ten or less). The examination of configuration histograms per element between repositories can help to identify such cases and also suggest ways to improve consistency within a given chemical collection.

In a more general view across PubChem and without restrictions of considered atom types, a total of 6,583 atom types [of which $2591(39.4 \%)$ are singletons] are unique to Substance and do not occur in Compound. The overall top-10 most frequent cases are presented in Fig. 12a. The reasons why they are not found in Compound are similar. In the case of types (i), (iv), (v), (vi), (viii) and (x), one of the covalent single bonds can represent a non-covalent interaction that is converted to a 
non-standard bond type during PubChem standardization. In addition to that, if all bonds implied in types (i) and (vi) are covalent, the hydrogen atom is removed and a positive charge is placed on the nitrogen atom. Type (ii) frequently occurs in nitro groups with two covalent double bonds to nitrogen. In that case, one of the double bonds is modified to be charge separated. In other cases, analogous to types (v), (viii) and ( $\mathrm{x}$ ), if all bonds are considered to be covalent, the corresponding substance is rejected during standardization. Type (iii) represents a nitrogen radical that is not in the PubChem "allowed" valence list. In the case of type (vii), the covalent single bond to yttrium is converted to a non-standard 'complex' bond. Mono-connected mono-valent argon is not in the PubChem allowed valence list, and no standardization rule for this case exists. Consequently, substances containing type (ix) are rejected during standardization.

There are only 31 atom types in Compound that are not found in Substance. The top-10 most frequent examples are shown in Fig. 12b. The first case is a result of the phosphorous atom being part of a non-covalent interaction (complex bond), where the charge is set during structure standardization. Analogously, the configurations found in the examples ranked second, third, fourth, sixth, seventh, eighth and ninth occur when trying to find adequate representations for complex bonds the respective atoms are incident to. The full list of atom types with their respective incidence is provided for Substance and Compound as supporting information in Additional file 3.

\section{Atom environment $(r=1)$ statistics}

The total number of atom environments with radius $r=1$ is 299,609 for Substance [100,411 (33.5\%) singletons] and 109,306 for Compound [31,163 (28.5\%) singletons], a $38 \times$ and $69 \times$, respective, fold increase over $r=0$. The distribution of fragment incidences is presented in Fig. 13. In both databases, respectively, only two atom environments with radius $r=1$ occur in more than $50 \%$ of all structures. The top-10 most frequent a

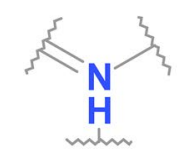

i) 206,162<smiles>CC(C)NC(C)C1C2CC3CC(C2)CC1C3</smiles>

vi) 35,998

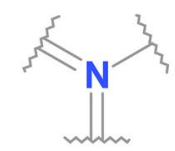

ii) 114,474

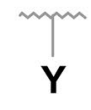

vii) 27,289

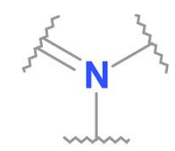

iii) 44,323

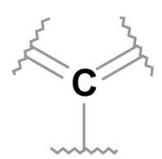

viii) 24,439

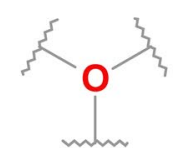

iv) 42,848

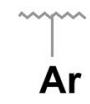

ix) 19,371

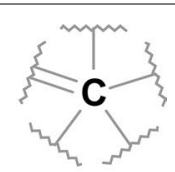

v) 36,600

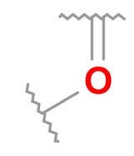

х) 19,129
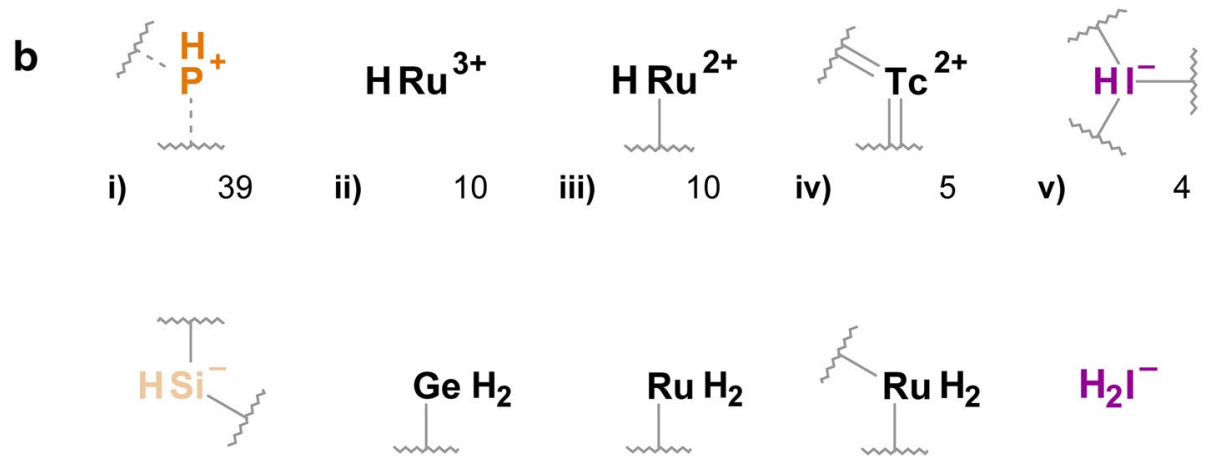

iii)

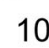

iv)

5

v)

4

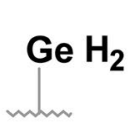

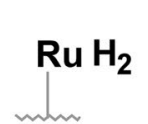

3
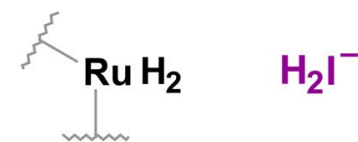

ix)

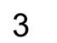

x)

3

Fig. 12 Top-10 most frequent atom types $(r=0)$ unique to Substance and Compound, respectively. Atom types are ranked by their incidence in the respective database: a Substance, and $\mathbf{b}$ Compound. Incidence provided in absolute numbers due to very low corresponding percentages.

Please note that $\mathbf{b}(i)$ is aromatic phosphorus and $\mathbf{b}$ (vii) is annotated as a monoradical. 
atom environments with radius $r=1$ in Substance and Compound are presented in Fig. 14. There is a clear indication that PubChem is dominated by structures containing aromatic fragments. While the atom environment consisting of three unbranched carbon atoms in a conjugated system is present in just above $50 \%$ of all structures (ranked second in both Substance and Compound), the corresponding branching atom environments are ranked first and third with examples shown in Fig. 14a (viii, ix) and $\mathrm{b}$ (viii, ix, $\mathrm{x}$ ). In both Compound and Substance, the ten top-ranked atom environments consist of carbon, nitrogen and oxygen only.

At least two functional groups are notorious for their variability and diverging preferred chemical structure representation: the azide and the nitro. To exemplify their heterogeneity, we performed a detailed analysis of the peculiarities of their basic element topologies present in PubChem. 'Azide-like' atom environments $\left({ }^{\prime *} \sim \mathrm{N} \sim \mathrm{N} \sim \mathrm{N}\right.$; ; di-coordinated nitrogen connected to a mono-coordinated and another di-coordinated nitrogen atom that is connected to some other atom) are found in Substance in 71 different variations of charge, hydrogen counts and connectivity as displayed in Fig. 15, of which 24 (34\%) are singletons. The general definition of 'azide-like' groups retrieves numerous cases where it is unclear whether they were actually meant to represent an azide group. However, the two resonance structures of the azide functional group are the most frequent examples, occurring in 118,976 and 7,983 substances, respectively. The non-charge-separated representation is the fifth most frequent atom environment, occurring in 512 substances. In Compound, the number of atom environments matching the general pattern of an 'azidelike' group is reduced to 37 [11 (29.7\%) singletons] with 6 new configurations that do not occur in Substance. Here, too, both resonance structures of the azide functional group exist, as normalization of azide functional group resonance structures was previously not handled by the PubChem structure standardization protocols. However, the non-charge-separated variant (fifth most frequent in Substance) is transformed to the most frequent atom environment of this type during PubChem

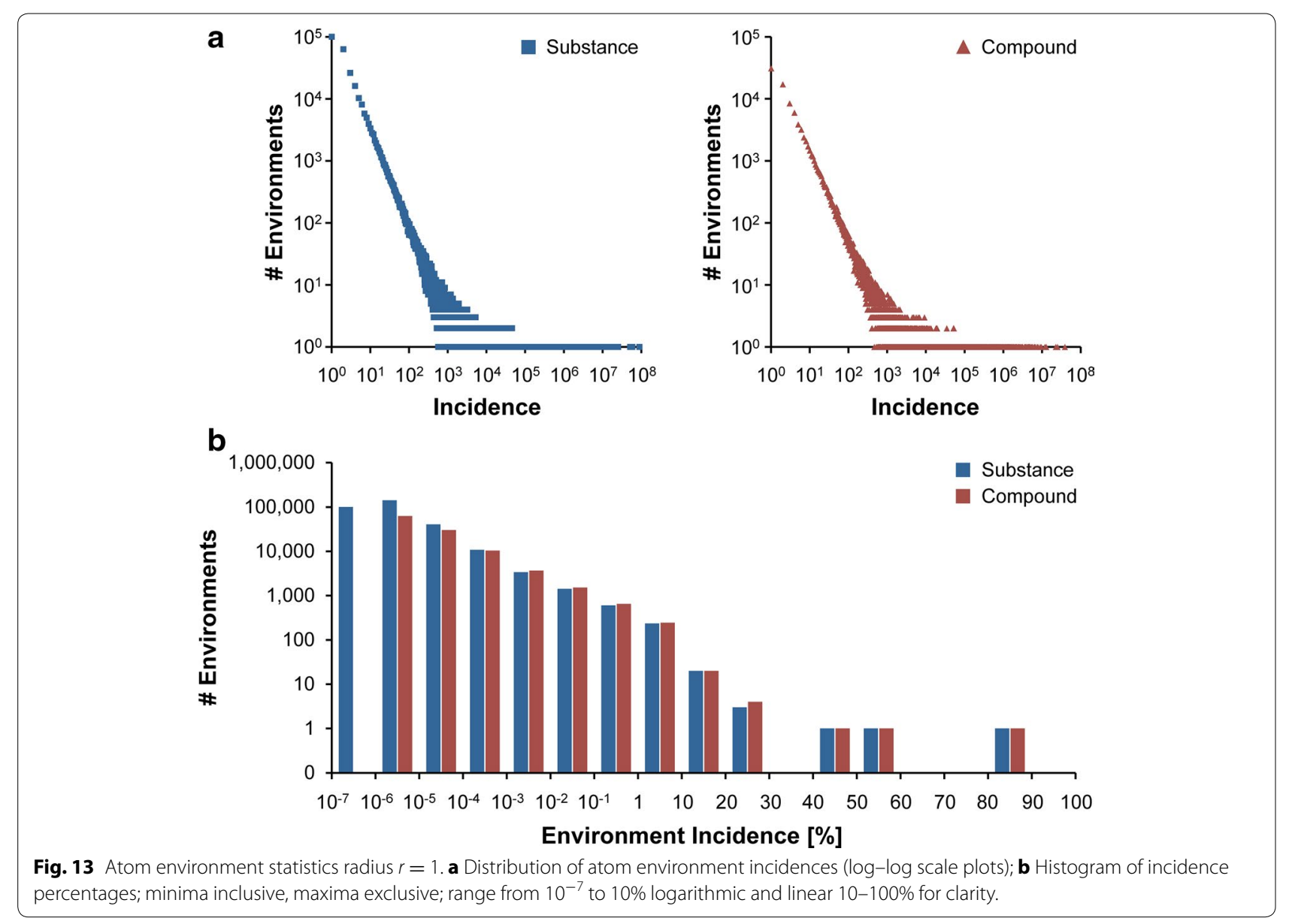


a

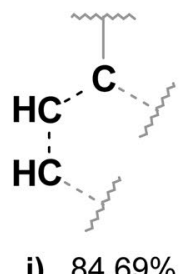

i) $84.69 \%$<smiles>CC=C(NC(C(C)C)C(C)C)C(C)(C)C</smiles>

vi) $22.72 \%$

b

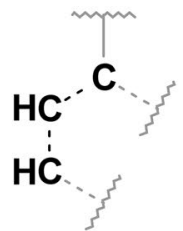

i) $82.85 \%$<smiles>CC(C)CC1CCCCC1C(C)(C)C</smiles>

vi) $25.10 \%$

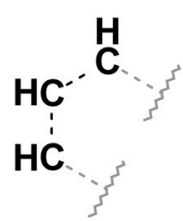

ii) $55.70 \%$

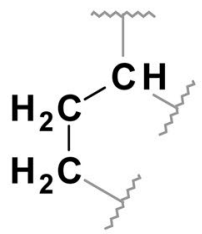

vii) $19.51 \%$

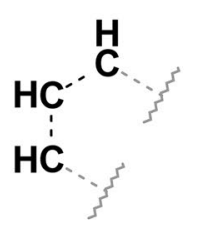

ii) $53.29 \%$

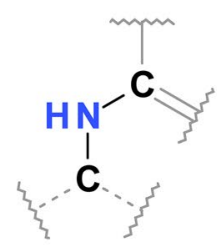

vii) $20.22 \%$

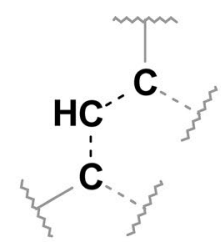

iii) $48.90 \%$

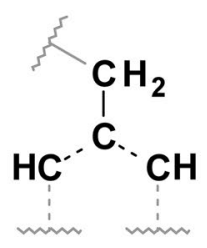

viii) $18.25 \%$<smiles>CC1CCCCC1</smiles>

iv) $23.99 \%$<smiles>CCCC(C(C)C)C(CC(C)C)OCC</smiles>

ix) $18.02 \%$<smiles>CCN1CCCC(C)(C)C1</smiles>

v) $23.90 \%$<smiles>CCC(C)C(NC(C(C)C)C(C)C)C(C)C</smiles>

x) $17.37 \%$

Fig. 14 Top-10 most frequent atom environments with radius $r=1$ in PubChem. Percentages indicate incidence of the atom environment in the respective database: a Substance, and $\mathbf{b}$ Compound. Light gray structures clarify valence and connectivity. Dashed lines indicate aromatic bonds as perceived using the aromaticity model OEAroModelOpenEye in the OpenEye Scientific Software, Inc. OEChem C++ toolkit [57].

standardization processing and therefore doesn't exist in Compound.

'Nitro-group-like' atom environments $\left({ }^{\prime *} \sim \mathrm{N}(\sim \mathrm{O}) \sim \mathrm{O}\right.$ '; tri-coordinated nitrogen atom connected to two monocoordinated oxygen atoms and some other atom) occur in 464 different variations of charge and connectivity, of which 148 (32\%) are singletons. If the atom type of the fourth 'other' atom is ignored, the number of variations is reduced to 60 as displayed in Fig. 16, with 13 (22\%) singletons. In Compound, this particular element topology occurs in 160 configurations [34 (21\%) singletons], a number that is reduced to 17 variations (zero singletons) if the atom type of the fourth, not necessarily terminal, atom is ignored.
There are a number of reasons why, for a particular atom environment, the number of structures it is incident to varies between Substance and Compound. If Substance contains a number of duplicates of a structure, each duplicate contributes to the atom environment incidence; however, this redundancy is not present in Compound, as it contains only unique structures in terms of valence bond structure representations as normalized by PubChem. PubChem standardization can modify an atom environment, decreasing the number of times the pre-standardization environment is present in Compound, or even eliminating it in favor of an alternative, but equivalent, representation. If a Substance chemical structure contains invalid or erroneous atom environment configurations 


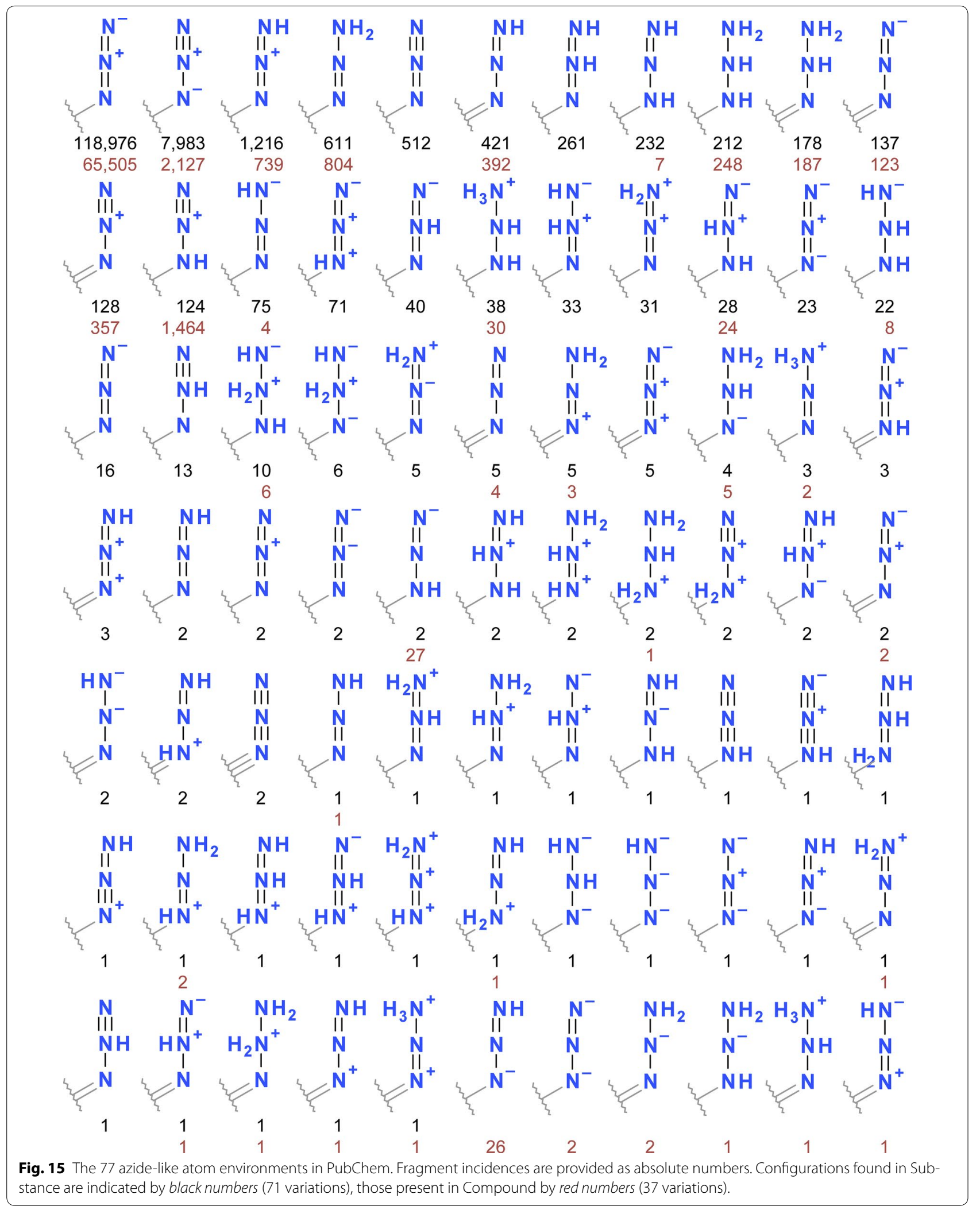




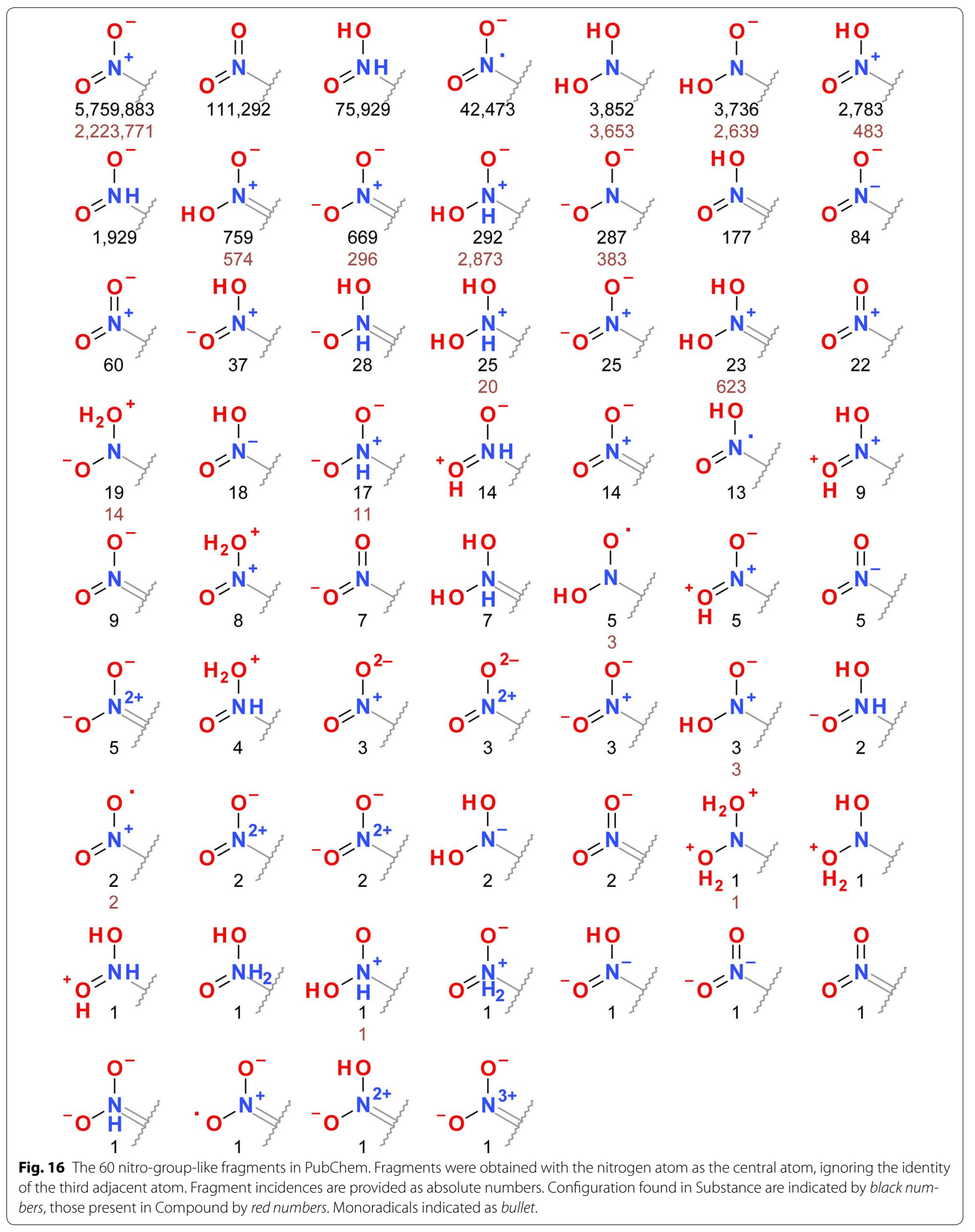


that cannot be salvaged by standardization, the corresponding substance structure is rejected and not present in Compound. Due to these effects, in general, the number of encountered atom environment variations and respective frequencies of occurrence will be reduced in Compound.

In total, 203,232 atom environments with radius $r=1$ occur in Substance but not in Compound, of which $81,702(40.2 \%)$ are singletons. The top-10 most frequent occurring of these atom environments are shown in Fig. 17a. The Substance unique atom environments of rank one, two and eight are instances of the generalized 'nitro-group-like' environments that are modified to the preferred representation of this functional group. The third and seventh most frequent $(r=1)$ atom environments unique to Substance are examples of modifications occurring during structure standardization that are due to tautomeric preference: the " $[\mathrm{OH}] \mathrm{C}=\mathrm{N}$ " pattern is modified to " $\mathrm{O}=\mathrm{C}[\mathrm{NH}]$ ", and the " $[\mathrm{NH}]-: \mathrm{C}=\mathrm{N}$ " pattern to " $\mathrm{N}=: \mathrm{C}-[\mathrm{NH}]$ " (where '-:' indicates an aromatic single bond and ' $=$ :' indicates an aromatic double bond). In the case of the atom environments ranked fourth, sixth, and tenth, depending on whether all indicated bonds actually represent covalent bonds, either the implicit hydrogen atom on the nitrogen atom is replaced by a positive charge, or it is removed when the further outgoing single bond is replaced by a non-standard bond type. Environments of ranks five and nine are related to the top ranked representation of the nitro group in a<smiles>CCC(C)C(C(C)C)[N+](=O)[O-]</smiles>

i) 97,438<smiles>CC(C)=NC(CC(C)C)C(C)C</smiles>

vi) 45,873

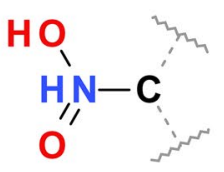

ii) 74,283

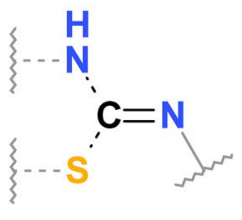

vii) 44,192

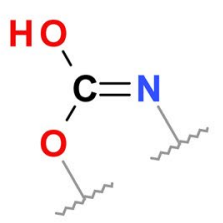

iii) 68,901<smiles>CC(C)C(C(C)C)N([O-])O</smiles>

viii) 42,202

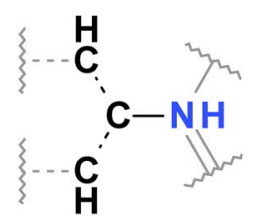

iv) 49,369<smiles>[Y]CC(C(C)C)N(CC)C(C)C(C)C</smiles>

ix) 41,280<smiles>[Y]CC(C=[V])N(CC)C(C)=CC</smiles>

v) 49,275<smiles></smiles>

x) 34,781<smiles>CC=C(C(C)C)[P+](O)(O)O</smiles>

i) 455<smiles>C[Si](C)=[14CH+]</smiles>

ii) 341<smiles>[Z7]=C(C(C(C)C)C(C)C)[C@@H](C)C(C)C</smiles>

iii) $\quad 339$<smiles>C=C(C)[P+](=C)C(=O)C(C(C)C)C(C)(C)C</smiles>

iv) 325<smiles>C[CH-]N(C(C)CC)C(C(C)C)C(C)C</smiles>

v) 266<smiles>CCOC1(OC(C)C)C(C)=CC2=[N+]1C(C)(C)CC2</smiles>

vi) 266<smiles>CC(C)[C-](C(C)C)C(C(C)C)[NH+]([C-](C(C)C)C(C)C)C(C)C</smiles>

vii)<smiles></smiles>

viii)<smiles>C=CC=[W]</smiles>

ix) 212

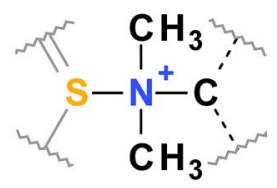

x) 211

Fig. 17 Top-10 atom environments with radius $r=1$ unique to Substance and Compound, respectively. Atom environments are ranked by their incidence in the respective database: a Substance, and $\mathbf{b}$ Compound. Incidence provided in absolute numbers due to very low corresponding percentages. Dashed lines indicate aromatic bonds as perceived using the aromaticity model OEAroModelOpenEye in the OpenEye Scientific Software, Inc. OEChem $\mathrm{C}++$ toolkit [57]. 
diverging molecular contexts and reflect modifications to the respective nitrogen atom after standardization.

There are 12,929 atom environments with radius $r=1$ in Compound that do not occur in Substance, of which 6,134 (46.6\%) are singletons. Consequently, all of them are a result of the PubChem structure standardization protocols. The top-10 most frequent examples are presented in Fig. 17b. In most cases, they are due to simple atom type modifications. In the most frequent and fourth most frequent case, tetra-valent phosphorous that was deposited uncharged gets assigned a positive charge. The charges on zirconium in the second and third most frequent cases are the result of attempts to find an adequate representation of complex bonds the respective atoms are involved. In a similar fashion, this is how the negative charges on carbon atoms in the fifth and seventh example occur. A positive charge is placed on tetra-valent nitrogen that was deposited uncharged in the cases ranked sixth, seventh and tenth. The di-valent chlorine atom showcased in the eighth most frequent environment exclusively found in Compound was deposited without charge and is modified to erroneously standardize to this atom type. In the substances that correspond to the ninth most frequent example, the tungsten atom is part of a complex with covalent single bonds that were removed during standardization by converting them to a PubChem complex bond, yielding a new atom type and consequently a new atom environment.

The full list of atom environments with radius $r=1$ with their respective incidence is provided for Substance and Compound as supporting information in Additional file 4.

\section{Atom environments radius $r=2$}

The number of unique atom environments with radius $r=2$ is $5,453,889$ in Substance $(1,637,544$ [30\%] singletons) and 4,559,587 in Compound (1,782,077 [39.1\%] singletons), an $18 \times$ and $42 \times$, respective, fold increase over $r=1$. The distribution of fragment incidences is presented in Fig. 18. Unlike with $r=1$, and emphasizing the diversity of structures when more atoms are considered, no fragment occurs in more than $50 \%$ of the structures in either Substance or Compound. The respective top-10 most frequent atom environments, presented in Fig. 19, are identical between Substance and Compound; however, the order is varied. In Substance, the most frequent atom environment occurs in $40.1 \%$ of all substances. The same fragment occurs in $37.3 \%$ of all compound records. In general, the top-ranked atom environments at the $r=2$ level are the logical extensions to the most frequent atom environments at the $r=1$ level, and, therefore, have a substructure-superstructure relationship to those shown in Fig. 13. For example the atom environments in Fig. 19b (i-iii) ( $r=2$, PubChem Compound) are combinations of those generated with a smaller radius, shown in Fig. 13b (i) and (ii) ( $r=1$, PubChem Compound). Superstructures to the non-aromatic environment with radius $r=1$ are not in the top-10 most frequent environments with radius $r=2$. Evidently, the frequency of aromatic fragments is sufficient such that variations including the next sphere of atoms can be found in more structures than the most frequent non-aromatic atom environments. This can be observed when comparing the incidences of the most frequent environments with radius $r=1$ and $r=2$.

The top-ranked environments present in Substance but not in Compound (Fig. 20a) can be explained by two effects. One is the standardization of the nitro group, responsible for unitizing the representations indicated in cases (iii), (v), (vi), (vii) and (x). The second one is a structural modification due to tautomer standardization procedures. In the cases shown in Fig. 20a (i) and (ii), which are closely related and actually possible extensions of each other, originating from diverging central atoms, the " $\mathrm{N}=\mathrm{C}-\mathrm{OH}$ " fragment gets standardized to the keto-equivalent " $\mathrm{HN}-\mathrm{C}=\mathrm{O}$ ". The same applies to rank (iv). For ranks (viii) and (ix), the " $\mathrm{N}=\mathrm{C}-$ $\mathrm{NH}$ " pattern is modified to " $\mathrm{HN}-\mathrm{C}=\mathrm{N}$ " such that the double bond is in a ring. For the top-ranked atom environment found in Compound but not in Substance is provided in Fig. 20b (i), the modification occurring during standardization actually takes place partly outside of the atom environment, but can be seen in its entirety in the cases shown in Fig. 20b (vi) and (vii), where the complete tautomeric system is found in the atom environment. It depicts a 1,5-shift of a proton between ring nitrogen atoms. In the case of the atom environment in Fig. 20b (ii), the hydrogen atom was originally located on the other oxygen atom (another proton 1,5-shift). Atom environments shown in Fig. 20b (iii, v) and b (viii) are examples of simpler modifications resulting from the application of standardization protocols. For example, the positive charge on the nitrogen atom resulted from fixing a penta-valent nitrogen atom. The atom environment shown in Fig. 20b (iv) is analogous to Fig. 20b (ii). In the case of atom environment Fig. 20b (ix), a proton on a ring nitrogen atom is transferred in a 1,3-shift to another ring nitrogen atom. In the case of atom environment Fig. 20b (x), the nitrogen atom type is changed during standardization from tri-valent, tri-coordinated with one implicit hydrogen atom to tri-valent, di-coordinated with no implicit hydrogen atom due the proton being shifted to an atom outside of the environment. 


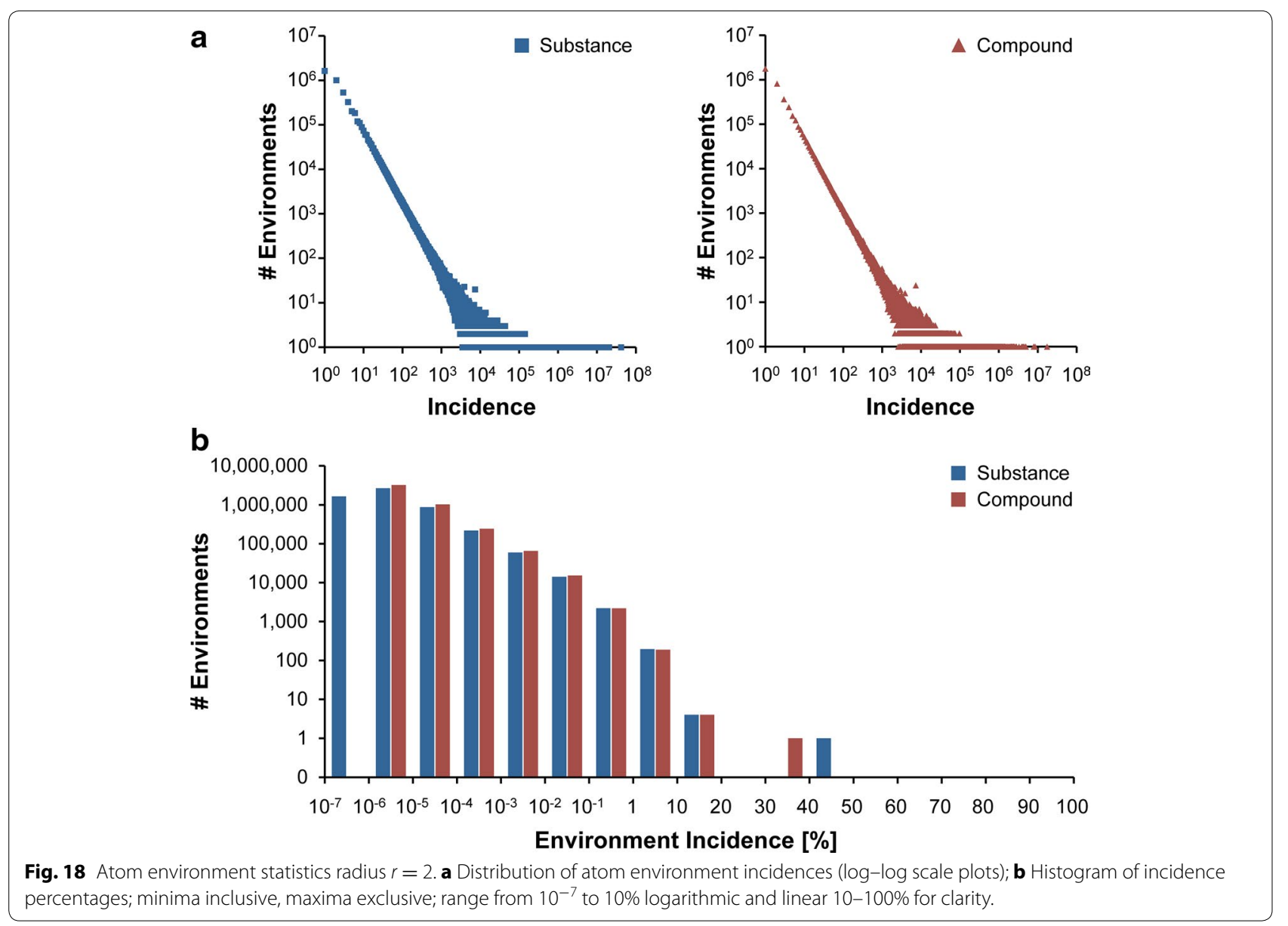

The full list of atom environments with radius $r=2$ with their respective incidence is provided for Substance and Compound as supporting information in Additional file 4.

\section{Atom environments radius $r=3$}

The number of unique atom environments with radius $r=3$ is $26,988,962$ in Substance $(7,879,785$ singletons, $29.2 \%$ ) and $25,115,177$ environments in Compound $(11,074,304$ singletons, $44.1 \%)$, a $4.9 \times$ and $5.5 \times$, respective, fold increase over $r=2$. The distribution of fragment incidences is presented in Fig. 21. The top-10 most frequent atom environments are presented in Fig. 22. The most frequent environment occurs in $19.5 \%$ of all substances and $18.3 \%$ of all compounds, respectively. In Substance, the most frequent atom environment with radius $r=3$ is a benzene-ring with only one substituent/attachment point (19.5\% incidence). In the second most frequent Substance atom environment $(r=3)$, this attachment is specified as a tetra-valent tetra-coordinated carbon atom with two implicit hydrogen atoms that is uncharged and not part of an aromatic moiety
(6.3\% incidence). It is a superstructure of the first ranked environment, the two differ by the center atom. Substance atom environments $(r=3)$ ranked third, fourth, fifth and sixth show examples with two substituents in the ortho position, where only in the case of the third ranked environment a connection to another aromatic system is indicated. In respective order of their ranks, these substituents are uncharged, aromatic, tri-coordinated and tetra-valent carbon with no implicit hydrogen atoms (5.4\% incidence), uncharged, tri-coordinated and tetra-valent carbon with no implicit hydrogen atoms ( $4 \%$ incidence), uncharged, di-coordinated and di-valent oxygen with no implicit hydrogen atoms (3.8\% incidence), and uncharged, di-coordinated and tetra-valent carbon with two implicit hydrogen atoms (3.8\% incidence). For these cases, the second substituent is not further specified, due to the location of the center atom of the environment; naturally, these generalized cases are more frequent than the more discriminative fragments specifying this part as well. The environments ranked seventh and eighth show examples for para substituted benzene rings, with one substituent being specified with only a 
a<smiles>[CH2]C/C=C\C</smiles>

HC. ${ }_{\mathrm{H}}^{-} \cdot \mathrm{CH}$

i) $40.08 \%$<smiles>CCC(C)NC(C)CCC(C)C</smiles>

vi) $9.75 \%$

ii) $19.58 \%$<smiles>CC1[CH][CH][CH][CH][CH]C1</smiles>

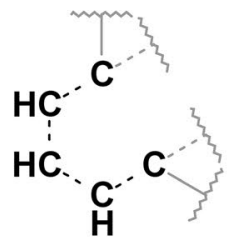

iii) $16.81 \%$<smiles>CCCCC(C)OCC</smiles><smiles>C/C=C(/CC)[C](C)[C](C)CC</smiles>

iv) $11.76 \%$

v) $10.83 \%$<smiles>CO[C@H](C)C(C(C)C)C(C)C</smiles>

HC<smiles>C[C@H]1CCC[C@H](C)C1</smiles>

vii) $9.32 \%$

viii) $9.31 \%$

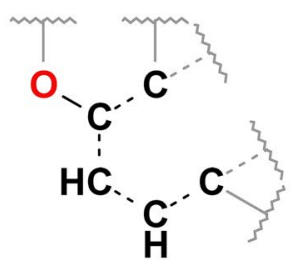

ix) $8.94 \%$<smiles>CCCCCC(CC)CCC</smiles>

x) $8.66 \%$

b<smiles>CCCCCCCCCC(C)C(C)C</smiles>

i) $37.27 \%$<smiles>CCCC(C)CCC(CC)C(C)C</smiles>

vi) $9.68 \%$<smiles>CC1[CH][CH][CH][CH][CH]C1</smiles>

ii) $18.39 \%$

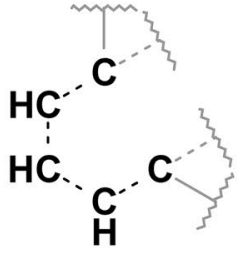

iii) $16.96 \%$<smiles>CCCC(CC)C(C)CC</smiles>

iv) $10.61 \%$<smiles>CC=C(C)C(C)CCC(C)C(C)C</smiles>

v) $10.52 \%$<smiles>CCC(C)NC(C)CCC(CC)C(C)C</smiles>

vii) $9.08 \%$<smiles>CCCCCC(C)CCC</smiles>

viii) $8.78 \%$<smiles>COCC(C(C)C)C(C)C</smiles>

HC<smiles>C1CC2CCC1C2</smiles>

ix) $8.28 \%$<smiles>CO[CH]C1C(C)CC2CC21</smiles><smiles>CC[CH][C]1[C]CCC1</smiles>

Fig. 19 Top-10 most frequent atom environments with radius $r=2$ in PubChem. Percentages indicate incidence of the atom environment in the respective database: a Substance; and $\mathbf{b}$ Compound. Light gray structures clarify valence and connectivity. Dashed lines indicate aromatic bonds as perceived using the aromaticity model OEAroModelOpenEye in the OpenEye Scientific Software, Inc. OEChem C++ toolkit [57].

single terminal chlorine atom (ranked seventh, $3.8 \%$ incidence) or a methyl group (ranked eighth, $3.7 \%$ incidence). The ninth- (3.7\% incidence) and tenth-ranked (3.7\% incidence) environments are closely related to the one with radius $r=2$ shown in Fig. 19a (iv), further specifying the substituent, and, in the case of the tenth-ranked, the sixth carbon atom in the phenyl ring.

The top-10 most frequent environments $(r=3)$ in Compound are almost identical to those in Substance. The three top-ranked fragments are the same. Between 
a<smiles>[Y]C=CC(C=[C])[N+](=O)O</smiles>
i) 68,459
ii) 62,735
ii) 48,680
iv) 45,641
v) 41,971

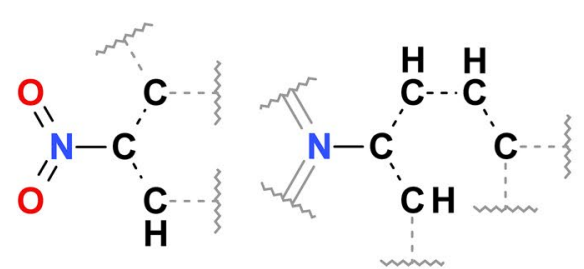<smiles></smiles>

vi) 40,957

vii) 35,141

viii) 34,481<smiles>CC=c1sc(=NCC)[nH]c1=CCC</smiles>

ix) 33,387<smiles>CC(C)=NC(C)C1CCC(C)CC1</smiles>

x) 31,844

b<smiles>CC=C(C)CC1C(=O)N=C(CC)N1NCC</smiles>

i) 2,982<smiles>CC(C)=C(C)C(C(=O)N1CCCC1)=C(O)C(C)C(C)C</smiles>

ii) 2,908

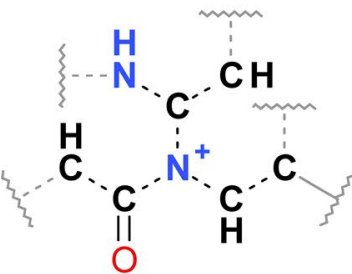

v) 1,585<smiles>CC(C)=C1N=C2C(C(C)C)=C(C(C)C)NN2C1C(C)C</smiles>

vi) 1,454 iii) 2,452<smiles>CCC(C)C(C=C(C(C)C)C(C)C)C(N)NC(C)C</smiles>

iv) 2,288<smiles>CCC(C)=C(C)C(C(=O)N1CCC1)=C(O)CCI</smiles><smiles>C=C1NC(C(NCC)C(CC)CC)NN1C(C)C</smiles>

vii) 1,454<smiles>CNC(NC)C(C=C(C)C)C(=O)[N+](C)(C)C</smiles>

viii) 1,444

ix) 1,335

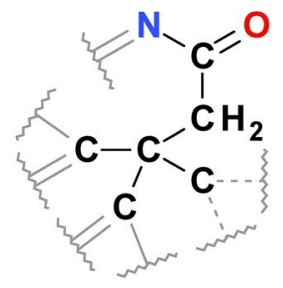

x) 1,322

Fig. 20 Top-10 most frequent atom environments with radius $r=2$ unique to Substance and Compound, respectively. Atom environments are ranked by their incidence in the respective database: $\mathbf{a}$ Substance; and $\mathbf{b}$ Compound. Incidence provided in absolute numbers due to very low corresponding percentages. Dashed lines indicate aromatic bonds as perceived using the aromaticity model OEAroModelOpenEye in the OpenEye Scientific Software, Inc. OEChem C++ toolkit [57]. 


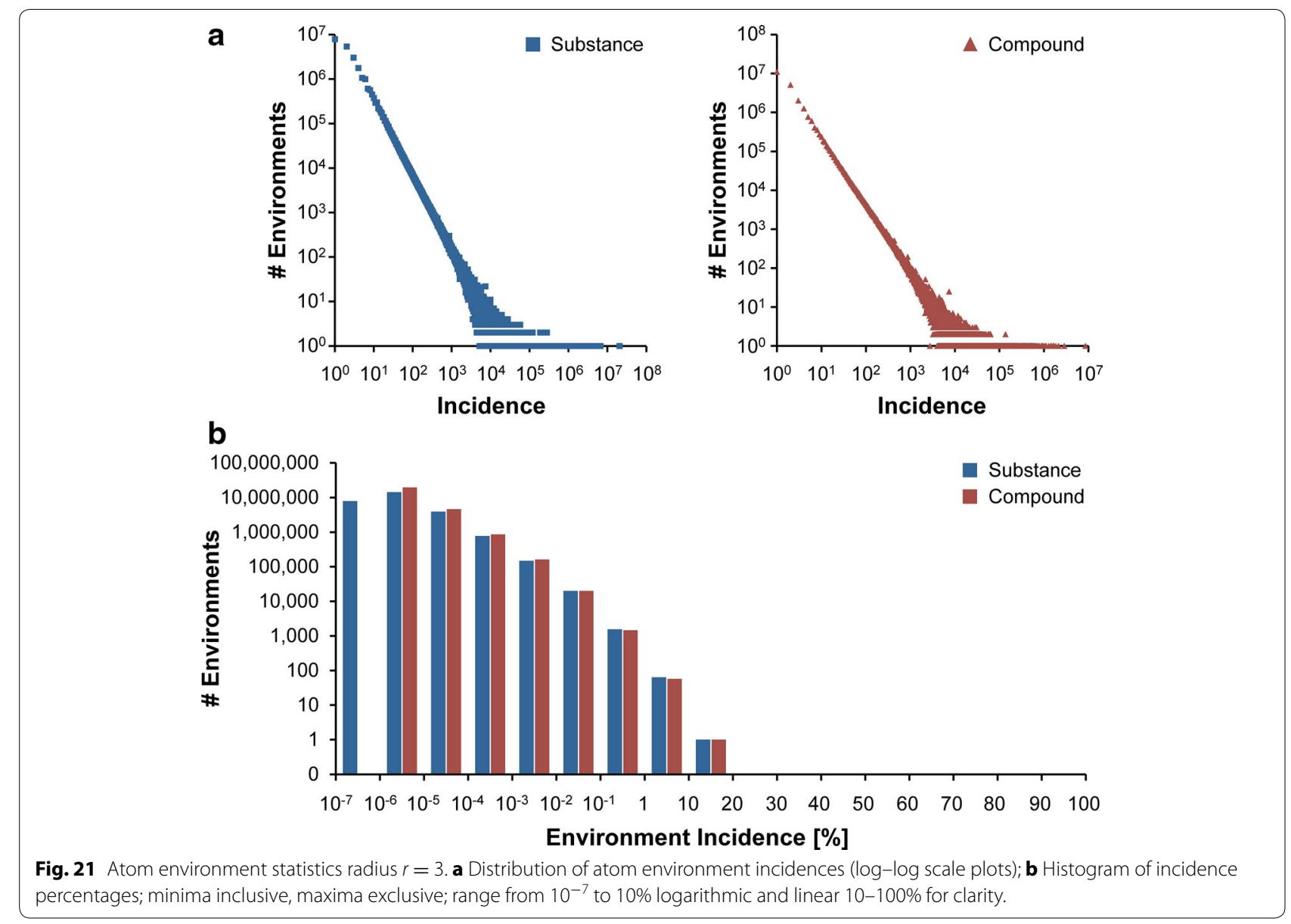

Substance and Compound, the fourth and sixth ranked environment switched ranks. Instead of a para substituted benzene ring with a terminal chlorine atom as one of the substituents, the fluorine case is more frequent in Compound and consequently ranked lower with 3.4\% incidence (fluorine, ranked seventh) compared to $3.1 \%$ incidence (chlorine, ranked ninth). The eighth ranked atom environment $(r=3)$ in Compound is not among the top-10 in Substance, with a tri-valent, tri-coordinated and uncharged nitrogen atom with one implicit hydrogen atom in ortho position to a second substituent in the benzene ring (3.1\% incidence). Lastly, the fragment with a methyl group in para position to a second unspecified substituent (3.1\% incidence) is ranked tenth in Compound compared to eighth in Substance.

The first and second most frequent atom environments $(r=3)$ that are found in Substance but not in Compound (Fig. 23) are closely related and show almost identical substructures originating from different central atoms. In both cases, during standardization, the proton from the exocyclic hydroxyl group is transferred in a 1,3-shift to the corresponding carbon atom, in the same step moving the double bond out of the five-membered ring. It is noteworthy that without the inclusion of all connecting bonds in the environment in the fragmentation model, the topranked environment is an example where the five-membered ring would not be closed. The next two cases, three and four, are similar as well, converting the "HO-C=N" fragment to " $\mathrm{O}=\mathrm{C}-\mathrm{NH}$ ". Cases five and six are again variations of the first two examples, one time with a different center atom than the environment ranked first, and the other time with a different aromatic carbon atom type. Fragments ranked seventh and ninth have different central atoms but exhibit the same 1,3-shift of a proton between nitrogen. The environment ranked eighth follows the same pattern as ranks three and four. In the last example listed in Fig. 23, during standardization the proton on the nitrogen atom is transferred in a 1,5-shift to its counterpart depicted at the top of the five-membered ring.

The atom environments $(r=3)$ identified to be present in Compound but not Substance (Fig. 24) are results of the PubChem standardization protocols. For the fragments ranked first and sixth, the deposited structure had the proton located on the nitrogen atom in the 
a<smiles>CC(C)(C)C1CCCCC1</smiles>

i) $19.54 \%$<smiles>CCCC1CCCCC1CCC(C)C</smiles>

vi) $3.81 \%$

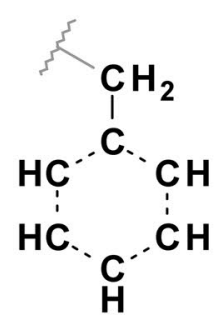

ii) $6.34 \%$<smiles>CC1CCC(Cl)CC1</smiles>

vii) $3.79 \%$<smiles>C[C](C(C)C)C1CCCCC1C</smiles>

iii) $5.38 \%$<smiles>C[C]1CCC(C)CC1</smiles>

viii) $3.67 \%$<smiles>CC(C)=C(C)C1CCCCC1C(C)C</smiles>

iv) $4.03 \%$<smiles>CCCC1CCCCCCC1OC</smiles>

ix) $3.66 \%$<smiles>CCOC1CCCCC1CC</smiles>

v) $3.84 \%$<smiles>CC[C@H]1CC[C@@H](OC)CC1</smiles>

x) $3.66 \%$

b<smiles>CC1C[CH]CCC1</smiles>

i) $18.34 \%$<smiles>CC(C)=C(C)C1CCCCC1C</smiles>

vi) $3.49 \%$<smiles>CC(C)CC1CCCCC1</smiles>

ii) $6.13 \%$<smiles>CC(C)(C)C1CCC(F)CC1</smiles>

vii) $3.44 \%$<smiles>CC(C)[C](C(C)C)C1CCCCC1C</smiles>

iii) $4.52 \%$<smiles>CCC1CCCCC1NC(C)C</smiles>

viii) $3.13 \%$<smiles>CC(C)CC1CCCCC1C(C)C</smiles>

iv) $3.95 \%$<smiles>CC(C)[C@H]1CC[C@@H](Cl)CC1</smiles>

ix) $3.11 \%$<smiles>CCOC1CCCCC1CC</smiles>

v) $3.56 \%$

Fig. 22 Top-10 most frequent atom environments with radius $r=3$ in PubChem. Percentages indicate incidence of the atom environment in the respective database: a Substance; and $\mathbf{b}$ Compound. Light gray structures clarify valence and connectivity. Dashed lines indicate aromatic bonds as perceived using the aromaticity model OEAroModelOpenEye in the OpenEye Scientific Software, Inc. OEChem C++ toolkit [57].

six-membered ring rather than the five-membered ring. The environments ranked second and eight similarly handle a case whereby the double bonded oxygen closest to the hydroxyl group originally was specified as hydroxyl group and the proton was moved during standardization (double bond changes implied). In the third ranked environment, analogous to the case shown in Fig. 20b (i) with $r=2$, a proton shift occurs that involves atoms in the original structure that are not part of this particular atom environment. Originally, the proton was located on the nitrogen atom that ends up being double bonded in the five-membered ring. Cases four and five involve an exocyclic double bond where the double bond was originally in the six-membered ring, showing a PubChem 


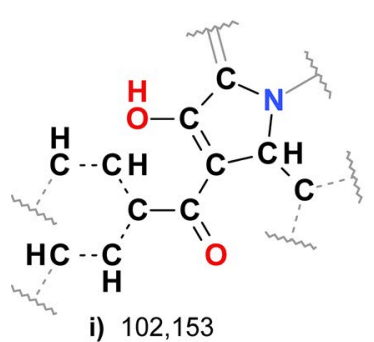

i) 102,153<smiles>CC(C)N=C(O)OC(C)(C)C</smiles>

iv) 62,733<smiles>[3H]CC(C[3H])C(=O)C1=C(O)C(=O)N(CCC)C1C(CC(C)C)CC(C)C</smiles>

ii) 75,577<smiles>CN=C(O)OC(C)(C)C</smiles>

iii) 68,459<smiles>CCCC1NC(=O)C(CCC)C(CC)N1</smiles>

vii) 49,481<smiles>[3H]CC(C[3H])C(=O)C1=C(O)C(=O)N(C(C)C)[C@H]1C(CCC)CC(C)C</smiles>

v) 55,522<smiles>CC=C1C(O)=C(C(=O)C(CC(C)C)CC2CCCCC2C)C(C(C)C)N1C(C)C</smiles>

vi) 53,259<smiles>CC(C)CC1NC(=O)[C@H]2CCCC[C@@H]2N1</smiles>

ix) 40,550<smiles>CC=C(C)C(CC)N=C(O)OC(C)(C)C</smiles>

viii) 45,629<smiles>CC=C(C)C1=C(C)NC2NC(C)NN2C1C(C)C</smiles>

x) 37,140

Fig. 23 Top-10 most frequent atom environments with radius $r=3$ unique to Substance. Atom environments ranked by incidence. Incidence provided in absolute numbers due to very low corresponding percentages. Dashed lines indicate aromatic bonds as perceived using the aromaticity model OEAroModelOpenEye in the OpenEye Scientific Software, Inc. OEChem C++ toolkit [57].

preference to put the tautomeric mobile proton on the nitrogen atom. The seventh-ranked environment is an example of a simple atom type modification, where the positively charged nitrogen atom was originally deposited uncharged with an additional (implicit) hydrogen atom attached. The last two (related) cases exhibit one completely observable modification and one that involves structural elements outside of the depicted fragment. The exocylic double bonded oxygen atom in the last case was deposited as hydroxyl group, but its proton got moved to the oxygen atom resulting as the hydroxyl group attached to the carbon atom that is part of the six-membered ring (double bond changes implied).

The full list of atom environments with radius $r=3$ with their respective incidence is provided for Substance and Compound as supporting information in Additional file 4 .

\section{Atom environment set overlap}

The sets of atom environments with radii $r=1, r=2$ and $r=3$ partly overlap, as illustrated in the Venn diagrams shown in Fig. 25. This overlap occurs for two 


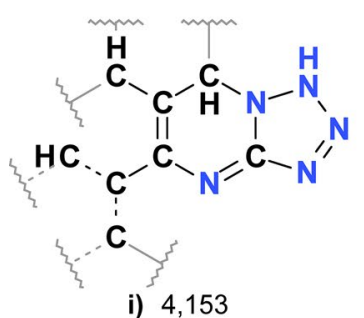

i) 4,153<smiles>CC=CC(CC)=C1C=CC=CN1</smiles>

iv) 2,863<smiles>CCN1C(=O)C(C#N)=C(C)C(=C(O)C(C)C)C1=O</smiles>

ii) 2,907<smiles>CC=CC(CCCC)=C1C=CC=CN1</smiles>

v) 2,857<smiles>CC1CC2=NC(=O)C(CC(=O)NC(C)C(C)C)N2N1</smiles>

iii) 2,893

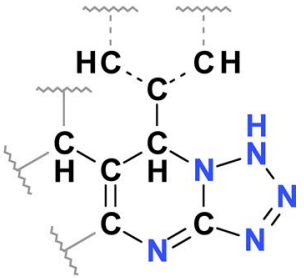

vi) 2,706<smiles>C/C=C\C1C(=O)[NH+]2CCCC(C)[C@H]2NC1C(C)C</smiles>

vii) 2,449<smiles>CC(C)=C1CC2CC(C(C)C)C(=C(C)C)C(=O)C2(O)C(O)=C1C</smiles>

ix) 2,200

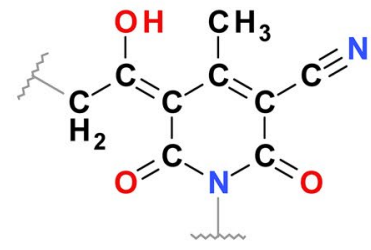

viii) 2,288<smiles>CC=C1C(=O)C2(O)C(O)=C(C(N)=O)C(=O)CC2CC1C</smiles>

x) 2,158

Fig. 24 Atom environments with radius $r=3$ unique to Compound. Atom environments ranked by incidence. Incidence provided in absolute numbers due to very low corresponding percentages. Dashed lines indicate aromatic bonds as perceived using the aromaticity model OEAroModelOpenEye in the OpenEye Scientific Software, Inc. OEChem C++ toolkit [57].

reasons. First, depending on the central atom and the molecular graph, starting from different atoms can result in the same atom environment with different radii. This is illustrated in Fig. 25c, using the example of dispiro(2.0.2.4)deca-1,5-diene (CID 143166). Second, atom environments can cover entire structures and consequently cannot grow any further with higher order radii. The second case is more prominent than the first one, with $13,918 / 43,575 / 916,758$ environments with radius $r=1 / r=2 / r=3$ describing complete structures in Substance, respectively. The corresponding numbers for Compound are 6,589 ( $r=1), 31,804(r=2)$ and 838,487 $(r=3)$. Naturally, there is no overlap between $r=1$ and $r=3$ environments exclusively. Such fragments would have to cover the entire chemical structure at $r=1$ level and therefore also be part of the set of environments generated for $r=2$. The top-10 most frequent cases for every radius in Substance and Compound are supplied as supporting information in Additional file 1: Figures S2 and S3.

\section{Atom environment rate of growth}

This atom environment survey of PubChem content is revealing for multiple reasons. One potential surprise is the high rate of singletons in Substance and Compound as a function of atom environment radius. The percentage of atom environment singletons for Substance is nearly constant, being $32.5,33.5,30$, and $29.2 \%$ for radius 


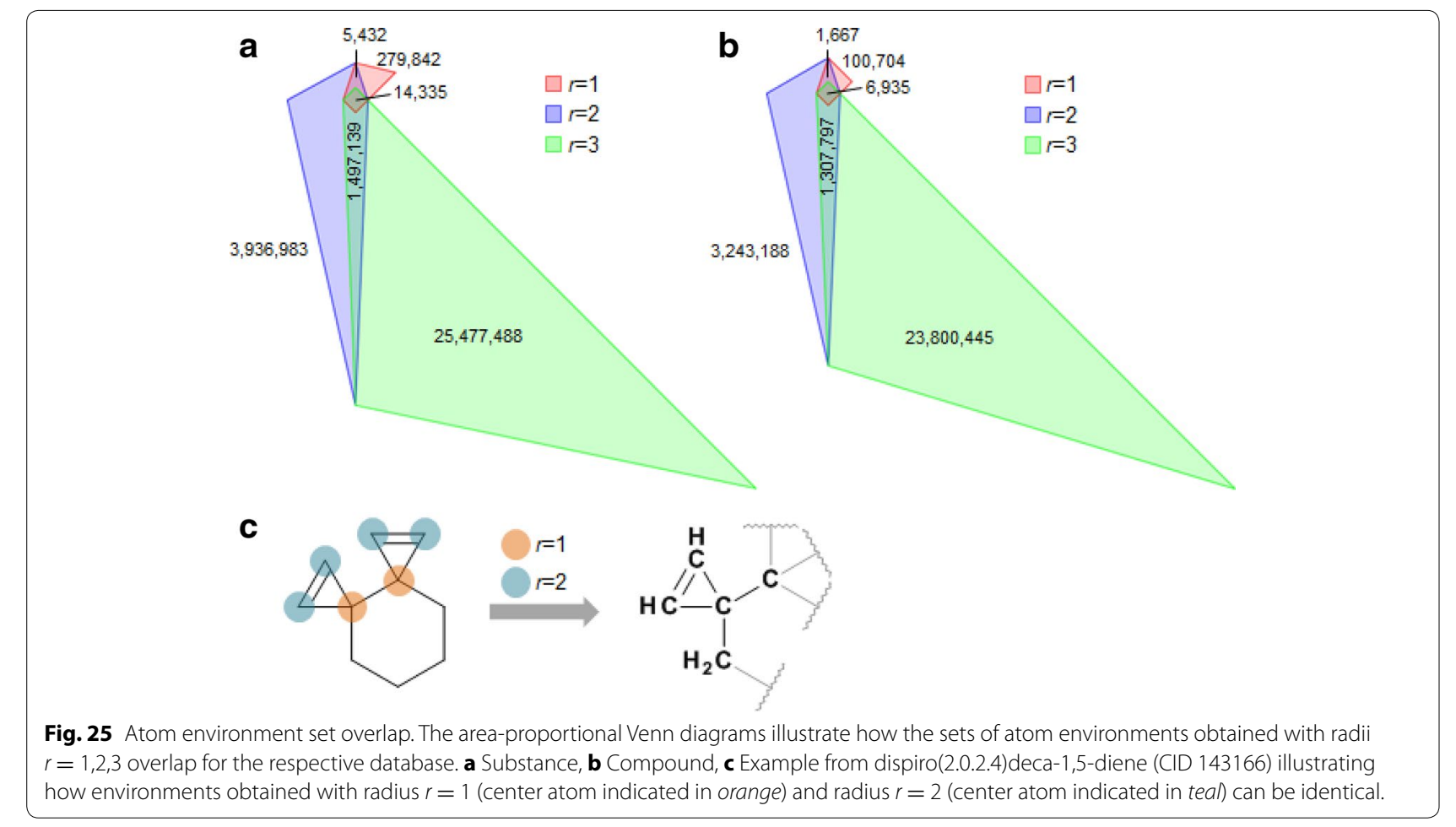

$r=0,1,2$, and 3, respectively. For Compound, however, the percentage of atom environment singletons is steadily increasing, being $10.6,28.5,39.1$, and $44.1 \%$ for radius $r=0,1,2$, and 3, respectively. Furthermore, the rate of growth of new atom environments in both Substance and Compound slows dramatically as a function of increasing radius, with an increase of 69, 42, and 6 times for Compound and 38, 18, and 5 times for Substance when considering the ratio of atom environment radius $0->1$, $1->2$, and $2->3$, respectively. The increasing quantity of singletons and the decreasing rate of growth of atom environments as a function of atom environment radius implies that there is still an enormous chemical space to explore even at the chemical fragment level, with nearly half of all $r=3$ molecular fragments in Compound appearing in only a single structure. It may also be surprising or revealing (to some) that the count of unique atom environments in Compound is so relatively few, being 1,583 ( $r=0), 109,306(r=1), 4,559,587(r=2)$, and $25,115,177(r=3)$. On the other hand, the drop in increase of number of fragments from $r=1$ to $r=2$ compared to $r=2$ to $r=3$ could also indicate that there are constraints limiting a full combinatorial exploration of the space defined by smaller fragments (e.g., steric effects). Furthermore, an increasing number of functional groups results in more reactive structures that would be increasingly harder to synthesize.
The high rate of singletons suggests that sampling a database for atom environments, such as in the earlier 1971 study, will miss the vast majority of atom environments present; however, sampling should be sufficient to locate common fragments. Therefore, the rate of growth of 'augmented atoms' determined in this study of 7.9 from 1971 CASRS to present day PubChem should be considered an upper bound due to the use of structure sampling in the 1971 CASRS study. Without the full CASRS database for comparison, a more accurate determination of rate of growth cannot be determined.

The concept of circular atom environments is the basis for so-called radial/circular [32, 33], Morgan [56], or extended connectivity fingerprints (ECFP) [34]. As feature ensemble fingerprints, they are not based on a predefined dictionary of structural features. However, we identified in total 28,460,736 unique atom environments of radii $r=1, r=2$ and $r=3$ (of which 12,470,387 are singletons, $43.8 \%$ ) in addition to 1,583 atom types ( $r=0$, 167 singletons, 10.6\%) in PubChem Compound, in total close to $2^{25}$ fragments. For the particular example of ECFP, where a hash function is used to generate 32-bit integers as identifiers for circular fragments [34], more than $99 \%$ of the available $2^{32}(4,294,967,296)$ bits in a fingerprint may remain unused. In Compound, the number of observed singletons increases with increasing radius, from $11 \%$ for atom types $(r=0), 29 \%$ at $r=1,39 \%$ at 
$r=2$, to $44 \%$ at $r=3$. It is unclear how well these singular differences between structures are captured in fixed size structural keys (such as those used by PubChem [72]). Conversely, as dynamically generated fingerprints like ECFP quantify structural differences including those previously unknown, this might be a possible explanation for their general advantage over other fingerprint approaches. Further analysis of molecular fragments, such as those provided in this study, may prove useful to design a better a set of discriminating (i.e., not co-linear) structural patterns useful for cheminformatic purposes such as structure searching (e.g., identity, substructure, similarity, etc.), virtual screening, clustering, clique analysis, and other conceivable applications.

\section{Atom environment errors}

This survey describes the content of PubChem and, as a consequence, has to deal with and is affected by its idiosyncrasies that can lead to an overestimation of atom types and, therefore, atom environments as well. Beyond automated checking by algorithms, there is no manual curation of data in PubChem, as it is not practical to curate tens of millions of chemical records; however, PubChem contains many manually curated chemical collections. Information is deposited into Substance and subjected to automated cleanup and standardization protocols, and the resulting standardized structures are the content of Compound. This only means that they are considered to be 'valid' chemistry, but not necessarily 'real' or 'correct' in terms of the intended structure. One can find cases where a 'valid' structure is in fact incorrect. For example, this can occur whenever something is mistaken for chemical information that has a different meaning in the original context. As shown in Fig. 26a, CID 60023123 contains radium (Ra), rubidium $(\mathrm{Rb})$ and yttrium $(\mathrm{Y})$ atoms. The structure, deposited as SID 142797524, originated from the USPTO patent application US2005165025. The Ra, Rb, and Y 'atoms' are actually placeholders to define larger moieties and are not really elements. From the same patent structure, the literals ' $\mathrm{X}$ ' and 'Rc', neither found in the periodic table, are not provided in this substance-instead they are provided to PubChem as carbon atoms. The same issue can be found with other literals used as placeholders in patents, but that also exist in the periodic table. In Fig. 26b, the case of rhenium ( $R e$ ) is illustrated with CID 60092055, found in SID 143170472 originating from USPTO patent US7335659. Rhodium (Rh) atoms can be the result of such a misinterpretation as well, as illustrated in Fig. 26c. The literal 'Rg' present in the original source of the same structure, USPTO patent US6835754, is not present in the structure provided to PubChem in SID 143366847, being replaced with a carbon atom.
Additional examples can be found for erroneously annotated rutherfordium (Rf) atom in CID 59869973, from SID 142371601, originating from USPTO patent US6214862 (Fig. 26d). There, fragments annotated as $R^{1}$ and $\mathrm{R}^{2}$ result in methyl groups in the deposited structure (again, due to replacement by a carbon atom). This structure contains a further example of a polonium (Po) atom that is annotated as a radical by PubChem standardization. Going back to the original patent, the 'Po' abbreviation indicates a protected hydroxyl group, but lost when the structure is added to PubChem. Another case of erroneous polonium atoms is exemplified in Fig. 26e. The structure in CID 60160982 (from SID 144104720) is labeled as 'polyacenaphthylene' by its depositor, which is more accurately represented as shown in Fig. 26e (iii). Apparently, in this case, 'Po' was originally used to indicate a polymer. Other abbreviations resembling atomic symbols that are used as abbreviations for fragments are U (uranium, CID 60130730, SID 143372400, USPTO patent US20040242832, Fig. 26f), V (vanadium) and W (tungsten, CID 60104420, SID 143231020, patent US6770648, Fig. 26g). In Fig. 26g (iii), the '-X' substituent that can be in 5- or 6-position in the ring as annotated is entirely lost in the deposited structure. Actinium (Ac) atoms can also be falsely annotated as shown in Fig. 26h (CID 60101345, SID 143213558, patent US6903102). There, the abbreviation 'Ac' was used to describe an acetyl group.

In order to estimate an upper bound for the number of atom types and atom environments possibly affected by the misperceptions outlined above, Table 3 provides counts for commonly mis-annotated elements. The total numbers of atom environments derived from the commonly mis-annotated elements for Substance are 679, $31,163,157,164$, and 298,715 for radius $r=0,1,2$, and 3, respectively. For Compound, the numbers are considerably lower, with $144,978,3,652$, and 6,980 , for atom environments with radius $r=0,1,2$, and 3 , respectively.

Even when considering only organic elements, erroneous structures consisting of valid atoms can be found in PubChem Substance and Compound, and will have an influence on atom environment diversity studies. Tetratert-butylmethane (Fig. 27a) is a hypothetical structure that has been identified as the smallest saturated and acyclic hydrocarbon that cannot be synthesized [73, 74]. Yet, it is present in Compound as CID 14123361 with the corresponding SIDs 137126462 and 143067637. Another example has been identified by Kolodzik et al. [75] as illustrated in Fig. 27b. The structure in CID 20695696 corresponds to SIDs 33924192 and 142503477 . The latter references USPTO patent US20020016531 as source, but no such chemical structure is specified in the patent, rather a chemical drawing package was used to represent 
<smiles></smiles>

i)<smiles>CC1CNCC(C)N1C</smiles><smiles>[R]N1C(C)CNCC1C</smiles><smiles>[R6]C=C(C)CC=[R18]</smiles><smiles>[R][Y]C(=O)c1c[Y]c2cc([R])c(N)cc12</smiles>

iii)<smiles>Cc1ccc2cc3c(c(I)c2c1)Cc1c[c-]ccc1-3</smiles>

$\operatorname{Re}$ i)<smiles>Cc1ccc2c(c1)Cc1c-2cc2ccc(I)cc2c1I</smiles>

ii)

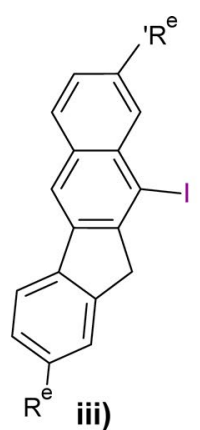

iii)
C<smiles></smiles>

i)<smiles>[O+]C1=Cc2cccc3cccc1c23</smiles>

i)<smiles></smiles>

ii)<smiles>[R9][N+]([O-])=Cc1cc(-c2ccccc2)c(O)c(-c2ccccc2)c1</smiles>

iii)<smiles>CC1C(=O)N2C(c3ccccc3)OCC2(C(Br)OP(=O)(O)OCc2ccccc2)C1C</smiles>

i)<smiles></smiles>

ii)<smiles>[R]C1C(=O)N2C(c3ccccc3)OCC(C([R])[R20])(C([R])[R20])C2C1[R]</smiles>

iii)

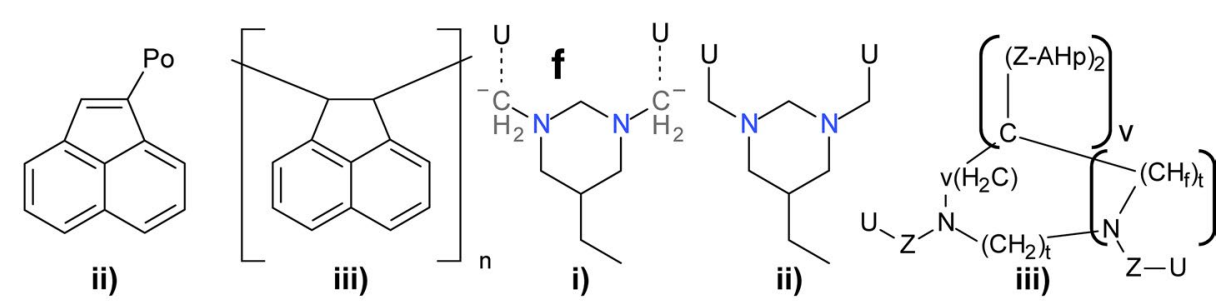<smiles></smiles>

i)<smiles>[Y]c1cc([N+](=O)[O-])cc(N)c1Sc1nc2ccccc2s1</smiles>

ii)<smiles>[X]c1cc2nc(Sc3c([Y])cc([N+](=O)[O-])cc3[Y])sc2c2c1CCC2</smiles>

iii)

h<smiles>CC(C)OC(c1cccs1)c1cccs1</smiles>

i)<smiles>OC(c1cccs1)c1cccs1</smiles>

ii)<smiles>CC(=O)OC(c1cccs1)c1cccs1</smiles>

iii)

Fig. 26 Sources of some erroneous atom environments in PubChem. a Radium (Ra), rubidium (Rb) and yttrium (Y), b Rhenium (Re), c Rhodium (Rh), d Rutherfordium (Rf) and polonium (Po), e Polonium (Po), f Uranium (U), g Vanadium (V) and tungsten (W), h Actinium (Ac). In all cases: $i$ as present in Compound, ii as deposited in Substance, iii as in the original context, except for (E), where it describes a correct way to annotate the 'polymer' aspect of the intended structure 'polyacenapththylene.' Charges and radical annotation (• doublet monoradical, $\wedge \wedge$ triplet diradical) in $i$ are a result of the PubChem standardization protocols. Dashed bonds indicate PubChem non-standard bonds.

a grid in a patent figure. The presence of these structures in PubChem is clearly erroneous. The use of atom environments may be an effective way to identify such 'valid' but implausible structures. For example, an atom environment with radius $r=1$ can describe tetra-tertbutylmethane, with a tetra-coordinated carbon with each adjacent atom being of the same type. Expanding such an atom environment to radius $r=2$, as illustrated in Fig. 27c, one can identify 247 entries in Compound containing this pattern. Taking this one step further, expanding the atom environment to radius $r=3$, as illustrated in Fig. 27d, yields 159 entries in Compound, a subset of those identified using the $r=2$ environment. After visual inspection, the 247 identified compounds appear to be 
Table 3 Statistics for elements potentially originating from misperceived abbreviations in PubChem Substance and Compound

\begin{tabular}{|c|c|c|c|c|c|c|c|c|}
\hline \multirow[t]{3}{*}{ Atomic symbol } & \multirow{2}{*}{\multicolumn{2}{|c|}{$\begin{array}{l}\text { Atom types } \\
r=0\end{array}$}} & \multicolumn{6}{|c|}{ Atom environments } \\
\hline & & & \multicolumn{2}{|l|}{$r=1$} & \multicolumn{2}{|l|}{$r=2$} & \multicolumn{2}{|l|}{$r=3$} \\
\hline & Sbst & Cmpd & Sbst & Cmpd & Sbst & Cmpd & Sbst & Cmpd \\
\hline V & 157 & 25 & 4,818 & 232 & 16,194 & 656 & 25,686 & 1,170 \\
\hline $\mathrm{Rb}$ & 28 & 4 & 1,388 & 7 & 9,054 & 9 & 19,098 & 12 \\
\hline Y & 80 & 10 & 7,432 & 24 & 57,213 & 47 & $1,16,554$ & 78 \\
\hline $\mathrm{Rh}$ & 102 & 21 & 3,133 & 71 & 9,231 & 122 & 14,561 & 167 \\
\hline W & 25 & 31 & 6,280 & 439 & 28,382 & 2,462 & 47,976 & 5,116 \\
\hline $\operatorname{Re}$ & 126 & 21 & 2,413 & 81 & 8,004 & 116 & 12,587 & 140 \\
\hline Po & 13 & 6 & 76 & 58 & 182 & 154 & 247 & 214 \\
\hline Ra & 12 & 3 & 1,527 & 7 & 11,355 & 9 & 24,073 & 9 \\
\hline Ac & 18 & 1 & 395 & - & 3,919 & - & 15,461 & - \\
\hline$U$ & 101 & 21 & 2,744 & 59 & 9,419 & 77 & 14,791 & 74 \\
\hline Rf & 17 & 1 & 957 & - & 4,211 & - & 7,681 & - \\
\hline
\end{tabular}

Elements are ordered by atomic number. For each combination of repository and radius, the number of unique fragments containing the respective atom element is listed. Substance and Compound are abbreviated as 'Sbst' and ' $\mathrm{Cmpd}$ ', respectively.

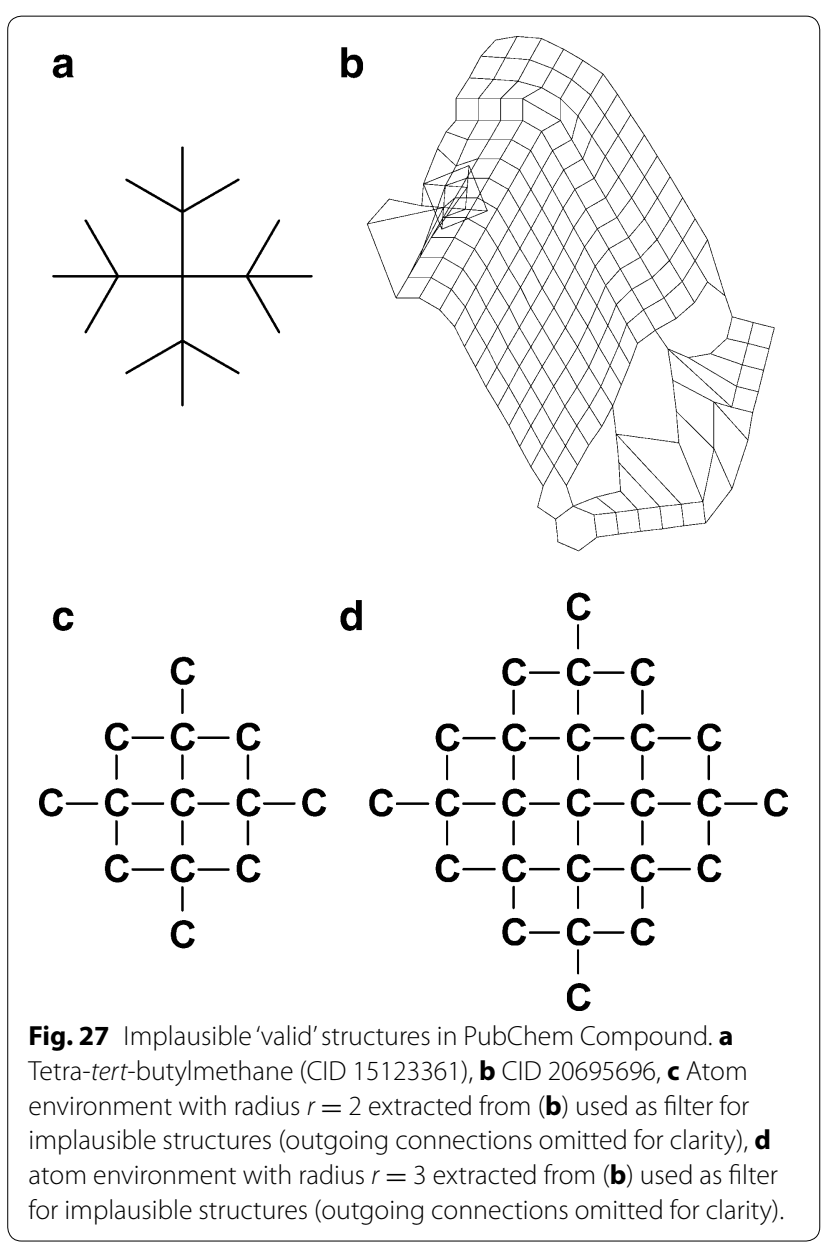

erroneous structures that do not represent 'real' chemicals. By selective manual examination of atom environments, one could generate filters to remove such classes of sterically implausible chemical structures.

These examples help to showcase that not all atom types and atom environments generated by this study resemble fragments of valid and real chemical structures. The belief is that these sorts of issues are rare and that they apply only to a relatively small number of atom types and atom environments. It also helps to demonstrate that simple atom environments (e.g., $r=0, r=1$, and beyond) are more tractable for manual curation than all of PubChem. Conceivably, these environments could be used as a 'sanity check' of real or plausible chemistry, and therefore are worthy of further investigation for chemical structure normalization and quality assurance (QA) purposes. The importance of this cannot be understated, as every new contribution to PubChem might exhibit structural elements previously unknown to the standardization protocols. A comparison of atom types and atom environments between Compound, Substance, and structures being contributed could automatically identify new representations and suggest structure examples for curation that can then be used for further refinement of standardization methods.

\section{Conclusions}

The chemical structure contents of the PubChem Compound and Substance databases was examined as a function of atom types and atom environments. The 
relative novelty of chemical structure fragments found in PubChem is considerable. The percentage of atom environments located in only a single PubChem Compound record is 10.6, 28.5, 39.1, and $44.1 \%$ for atom environment $r=0$ (atom type), 1, 2, and 3 (ECFP_6 like), respectively. Considering many chemical structures are synthesized for novelty purposes, this may not be completely surprising. Interestingly, the relative rate of increase of new atom environments, while still substantial, slows dramatically when examined as a function of increasing atom environment radius in PubChem Compound, with a 69,42 , and 6 -fold increase for $0->1,1->2$, and $2->3$, respectively. This suggests that there is still considerable room for chemists to pursue novel chemical structures using only new combinations of smaller (e.g., $r=2$ ) atom environment fragments.

Further emphasizing this point, plots of the incidence of atom environment fragments at various sizes show a $\log / \log$ behavior. In some ways, this may suggest that chemists lack imagination in that the majority of chemical structures contain one or more of the same basic molecular fragments. One could also easily argue the opposite point, in that chemists are constantly pushing into new and unexplored areas of chemistry and are rarely using the same atom fragments twice. In the end, it seems very clear that chemists have plenty of room to explore new and sparsely explored chemistry space and, therefore, make many new discoveries for some time to come.

The analysis of PubChem Compound was compared to similar studies performed over 40 years ago by Crowe et al. and Adamson et al. of CASRS chemical structures. A near eightfold $(8 \times)$ increase in $r=1$ atom environments ('augmented atoms', atom and its nearest neighbors) was found. While this result can only be considered an upper bound, due to the use of structure sampling by the earlier studies and the relatively high rate of singletons found in the PubChem analysis, it does imply a substantial increase in the capability of chemists to synthesize and isolate novel chemistry as a function of time, with a noted increase in the prevalence and popularity of nitrogen and oxygen containing atom environments now as opposed to then. The supporting information provided in this study should allow for future comparisons on the progress and trends of chemists.

The differences between the PubChem Substance and Compound databases were examined, in part, by using examples of atom environments of increasing size unique to each repository. This study noted the count of unique atom environments in Substance is greater than it is in Compound. This is due to the fact that structures in Substance undergo structure standardization and have to pass validity filters before becoming part of Compound.
This 'sanity' step dramatically reduces the count of atom environments by removing implausible chemistry (e.g., five bonds to carbon) and by normalizing varying functional group representations. These differences also help to emphasize the effect of PubChem standardization protocols for preferred atom types and particular tautomeric/resonance forms such that they could be used as the basis for a fragment-based structure normalization procedure.

The analysis of the Compound database is particular helpful to understand and characterize the diversity of molecular fragments found in known chemicals. Given the limited number of atom environments up to $r=3$ (ECFP_6 like), it may be possible to do a more thorough examination of observed fragments to improve the efficiency of chemical information algorithms, such as those for chemical structure searching or virtual screening. Furthermore, the results of this study highlight that further refinement of standardization procedures in PubChem will be beneficial.

\section{Methods}

In this analysis, the OpenEye Scientific, Inc. OEChem $\mathrm{C}++$ toolkit was used for the representation of atoms, bonds, and molecules [57].

\section{Structure preprocessing}

Most standard formats for structure representation in chemical information, such as SMILES [11, 12] and connection table file formats [23-25] do not require the specification of explicit hydrogen atoms in a chemical structure or implicit hydrogen atom counts. Instead, a standard valence model is employed, where implicit hydrogen atom counts are determined from (among other things) the atomic number, explicit atom valence and formal charge. Standard valence models can vary between file formats and software implementations. In PubChem Substance, the presence of explicit hydrogen atoms are nearly always limited to chemical structures with a hydrogen atom involved in the configuration of a stereocenter or to specify a particular isotope form. Consequently, most non-hydrogen atoms in Substance have non-saturated valences, and the chemical structures do not represent valid chemistry without additional processing to assign implicit hydrogen counts. In order to account for these effects, Substance records were subjected to a standard valence model prior to atom environment analysis by invoking the OEChem $\mathrm{C}++$ toolkit [57] function OEAssignMDLHydrogens. PubChem Compound is derived from Substance through automated structure standardization protocols, including the adjustment of implicit hydrogen atom counts and subsequent assignment of explicit hydrogen atoms. For the purpose 
of this analysis, all explicit hydrogen atoms of substances and compounds were converted to implicit hydrogen atom counts using the OEChem $\mathrm{C}++$ toolkit [57] function OESuppressHydrogens with all Boolean parameters set to 'false'. Please note that this explicit-to-implicit hydrogen atom conversion removes all explicit hydrogen atoms, including those with specific hydrogen isotopes, affecting 98,342 deuterium and 21,039 tritium containing substances, as well as 56,725 deuterium and 8,909 tritium containing compounds, respectively.

\section{Atom types}

In this study, we employed two atom typing schemes. For an adequate comparison of fragments in PubChem Compound to the results of an 'augmented atom' study of the CASRS published by Adamson et al. [47], atoms are characterized by their atomic number as sole feature. For a more detailed analysis of circular atom environments in PubChem Substance and Compound, atoms are characterized by six properties: (1) atomic number; (2) formal charge; (3) implicit hydrogen count; (4) explicit degree; (5) valence; and (6) participation in a conjugated (aromatic) system. The atom "explicit degree" is the number of explicitly connected atoms. The atom "valence" equals the sum of all incident sigma and pi bonds. The number of "incident sigma bonds" is described by the sum of "implicit hydrogen count" and "explicit degree". The number of "incident pi bonds" is the sum of bond orders of explicitly connected atoms minus the "explicit degree". This atom characterization approach allows a description of the molecular context of an atom (environment) without having to include the next layer of atoms as pseudo atoms as in other approaches [76]. Atom aromaticity was perceived using the OEChem $\mathrm{C}++$ toolkit function OEAssignAromaticFlags in combination with the aromaticity model OEAroModelOpenEye. In the specific case of the comparison with CASRS, the OEAroModelMDL was used, as it allows for a more "apples to apples" comparison to the older study by allowing only six-membered rings of carbon and nitrogen to be aromatic, provided they satisfy the 'Hückel $4 n+2$ ' rule $[58,59]$ (i.e., atoms are $\mathrm{sp}^{2}$-hybridized).

\section{Bond types}

In this study, we employed two bond typing schemes. For an adequate comparison of fragments in PubChem Compound to the results of an 'augmented atom' study of the CASRS published by Adamson et al. [47], bonds are characterized by their covalent bond order (single, double, triple), and presence in ring or chain, plus an additional 'aromatic' ring bond type. Bond aromaticity was perceived using the OEChem $\mathrm{C}++$ toolkit [57] function OEAssignAromaticFlags in combination with the aromaticity model OEAroModelMDL. For a more detailed analysis of circular atom environments in PubChem Substance and Compound, four different bond types are distinguished: single, double, triple, and aromatic. Bond aromaticity was perceived using the OEChem $\mathrm{C}++$ toolkit [57] function OEAssignAromaticFlags in combination with the aromaticity model OEAroModelOpenEye. In addition to covalent bonds, PubChem defines and actively uses three non-standard bond types: ionic, complex and dative bonds. In this analysis, these non-standard bond types were completely ignored.

\section{Atom environments}

Atom environments combine atom types and bond types into larger fragments. In this study, we employed the concept of circular atom environments centered on a particular atom referred to as the center atom. An atom environment of radius " $r$ " contains all atoms in the molecule with a topological distance $r$ or smaller to the center atom [55], and all bonds between them. The topological distance between two atoms is measured as the number of bonds on the shortest path between them. Consequently, atom environments with radius $r=0$ include only the atom type of the center atom. Atom environments with $r=1$ contain the center atom, all atoms adjacent to the center atom (nearest neighbors), and all the bonds between these atoms (those connecting the center atom with its neighbors and those between the neighbor atoms, if any exist). The inclusion of all bonds between atoms in an atom environment facilitates better separation between atom environments in ring-close scenarios as illustrated in Fig. 28. The advantages of including aromaticity and connectivity information in atom and bond types are illustrated in Fig. 29.

Atom environments with $r>0$ were not generated with terminal atoms as center atoms, referring only to atoms that are adjacent to one other atom. These terminal atoms are included in the environment originating from the adjacent-non-terminal-partner. However, this exclusion of terminal atoms means that mono- and di-atomic structures are excluded from any atom environment analysis when $r>0$, as they consist exclusively of terminal atoms. In Substance, this leads to 1,797 mono-atomic and 3,795 di-atomic structures being excluded from the atom environment $r>0$ analyses. In Compound, this leads to 448 mono-atomic and 1,306 di-atomic structures being excluded from the atom environment $r>0$ analyses. Statistics for these excluded structures are provided in the supporting information. (See Additional file 1: Figures S4, S5) Terminal atoms are included in the atom environment $r=0$ (i.e., atom type) analysis.

In order to generate canonical representations for atom environments, we used functionalities from the OpenEye 


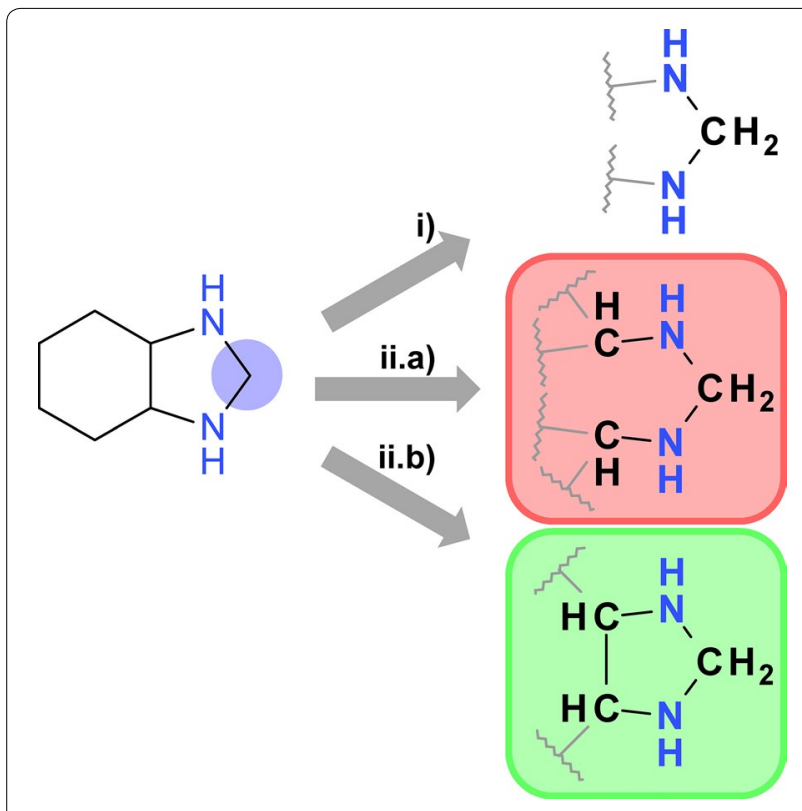

Fig. 28 Bond inclusion in atom environment generation. Atom environments contain all bonds between included atoms. The effect is illustrated using the example of perhydrobenzimidazole (CID 21866348), the highlighted atom is the center atom in this example. $i$ Atom environment with radius $r=1$, ii.a Atom environment with radius $r=2$ without including all connecting bonds, the five-membered ring remains open, ii.b atom environment with radius $r=2$, including all connecting bonds closes the five-membered ring and enables distinguishing this case from branching scenarios.
Scientific Software, Inc. OEChem C++ toolkit [57]. Atom environments were encoded as simplified molecular-input line-entry system arbitrary target specification (SMARTS) patterns [77]. For each atom in an OEMol object that represents a PubChem substance or compound record, a SMARTS representation was generated according to the encoding described in Tables 4 and 5 . This string representation of atom properties was set as the atom name by invoking the method SetName, after setting the atomic number to ' 0 '. Atom environments were programmatically generated as OEMol objects containing only the atoms of a molecule with a shortest topological distance to the center atom that is lesser or equal to the atom environment radius, and the bonds between those atoms. A canonical string representation of the OEMol object representing the atom environment was generated by invoking the function OECreateSmiString with the OESMILESFlag values 'Canonical' and 'SuperAtoms'. As the atomic number was set to '0', canonicalization uses the string value set as atom name for prioritization during the canonicalization procedure. These canonical representations of atom environments, as provided in Additional file 4, were the basis for our study.

\section{Incidence and occurrence}

In this study, atom environment frequency is expressed in terms of incidence and occurrence. Incidence refers

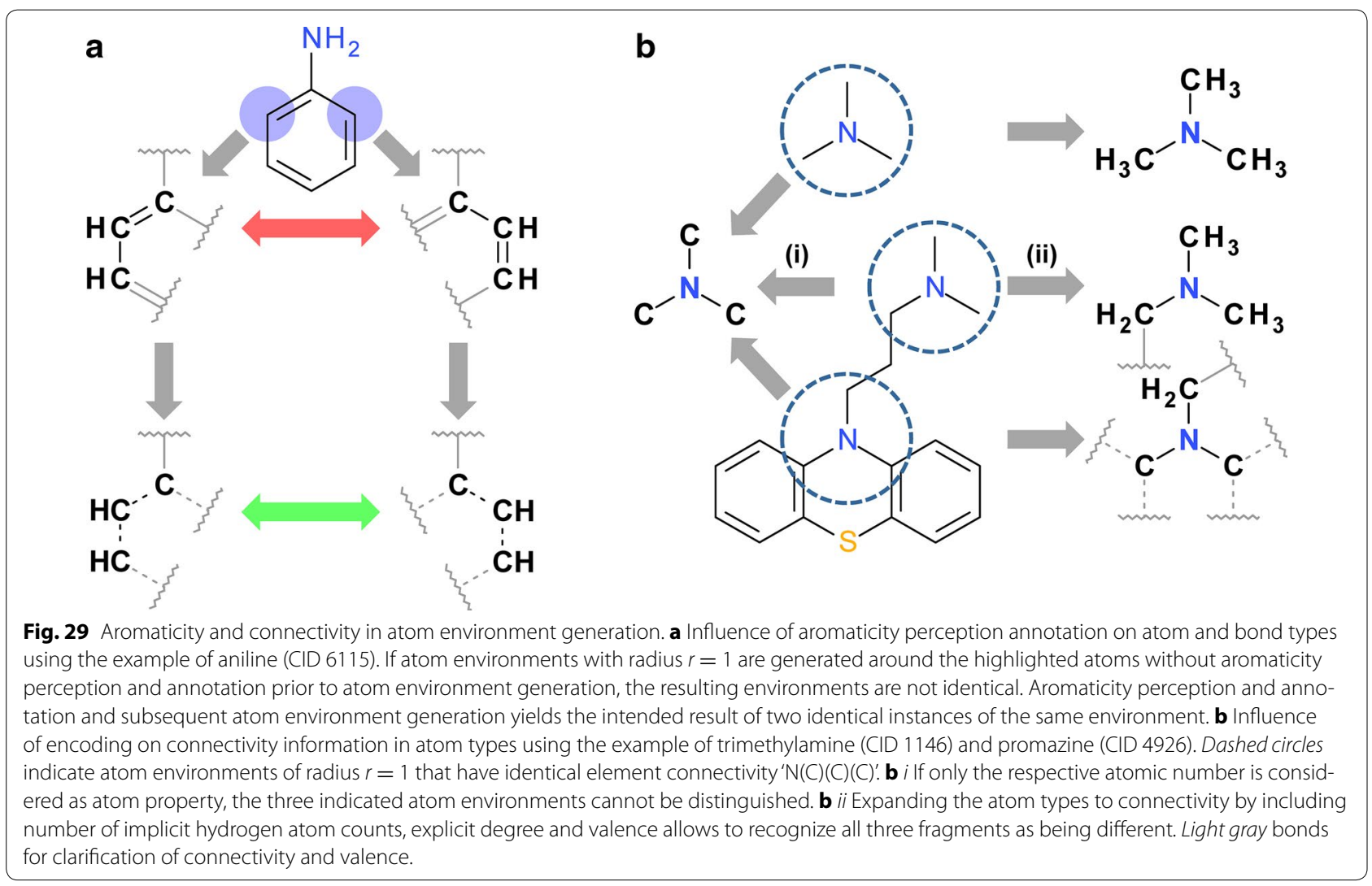


Table 4 Atom and bond primitives for encoding of 'augmented atoms' in SMARTS

\begin{tabular}{lll}
\hline Feature & SMARTS encoding & Special case \\
\hline Atom primitives & & \\
Element & \#<atomic number> & \\
Bond primitives & & \\
Single bond & - & $@(! @)$ for 'in ring' ('not in ring') \\
Double bond & $=$ & $@(! @)$ for 'in ring' ('not in ring') \\
Triple bond & $\#$ & $@(! @)$ for 'in ring' ('not in ring') \\
Aromatic bond & $:$ &
\end{tabular}

Encoding specific to the environment analysis performed in comparison to the results published by Adamson et al. [47].

Table 5 Atom and bond primitives for encoding of atom types and atom environments in SMARTS

\begin{tabular}{|c|c|c|}
\hline Feature & SMARTS encoding & Special case \\
\hline \multicolumn{3}{|l|}{ Atom primitives } \\
\hline Element & Atomic symbol & $\begin{array}{l}\text { Lower case indicating } \\
\text { aromaticity }\end{array}$ \\
\hline Formal charge & $\pm<$ integer $>$ & $\begin{array}{l}\text { Uncharged represented } \\
\text { as }+0\end{array}$ \\
\hline $\begin{array}{l}\text { Implicit hydrogen } \\
\text { count }\end{array}$ & $\mathrm{h}<$ integer $>$ & \\
\hline Explicit degree & $D<$ integer $>$ & \\
\hline Valence & $v<$ integer $>$ & \\
\hline \multicolumn{3}{|l|}{ Bond primitives } \\
\hline Single bond & - & \\
\hline Double bond & $=$ & \\
\hline Triple bond & $\#$ & \\
\hline
\end{tabular}

Aromatic bonds are implied between aromatic atoms unless explicitly specified otherwise.

to the absolute count or percentage of (substance or compound) records that contain a particular fragment. Occurrence refers to the absolute count or percentage of all fragments across all structures. Therefore, per chemical structure record, occurrence considers all fragments, while incidence considers only the unique fragments.

\section{Dataset}

This study uses PubChem as it existed on January 14, 2013 with maximum SID 160,655,685 and maximum CID $70,680,246$. For both data sets, only PubChem records searchable ('live') at that point in time were processed. PubChem Substance records with 'auto-generated' structures were excluded. In 'auto-generated' cases, no actual structure is deposited, but a reference to a PubChem Compound record is derived using chemical names and may include chemical name conversion using various approaches, including the OpenEye Scientific Inc. Lexichem $\mathrm{C}++$ toolkit [78]. Lastly, the chemical structure for a given substance had to be fully specified. Therefore, substances containing arbitrarily defined atoms (pseudoatoms) were excluded from this analysis. By these criteria, atom environments $(r=0,1,2,3)$ were determined for 104,669,789 Substance records. All 46,704,121 'live' records in Compound were also processed.

All atom environments $(r=0,1,2,3)$ found are provided as supporting information in Additional files 3 $(r=0$; atom types) and $4(r=1,2,3$; atom environments $)$ as SMARTS patterns. Usage of atom and bond primitives for encoding of augmented atoms and PubChem atom environments are detailed in Tables 4 and 5, respectively. Provided in this format, fragments can be visualized using appropriate techniques [79, 80], or readily imported into various toolkits. All SMARTS patterns supplied as supporting information have been tested for their validity by successfully parsing them through the OEChem C++ toolkit [57] function OEParseSmarts.

\section{Records}

Records may be referred to as SID (substance identifier) for PubChem Substance records and CID (compound identifier) for PubChem Compound records. Atom environments that occur in only a single PubChem record are referred to as singletons.

\section{Additional files}

\section{Additional file 1: Supplementary Figures and Tables.}

Additional file 2: PubChem Compound augmented atoms. This file contains all 'augmented atoms' generated from PubChem Compound for the purpose of comparison to the fragmentation studies of Adamson et al. performed on CASRS in 1971 [46]. Each line describes an augmented atom as SMARTS string and its respective incidence, tab-separated. File is sorted by descending incidence.

Additional file 3: PubChem atom types. This file contains all atom types generated in this study for PubChem Substance and PubChem Compound in SMARTS format. Each SMARTS string is followed by a semicolon-separated incidence vector that specifies in how many records in which repository the particular atom type was found. Order of repositories is PubChem Substance followed by PubChem Compound. File is sorted by lexicographical order of SMARTS strings.

Additional file 4: PubChem atom environments. This file contains all atom environments generated in this study for PubChem Substance and PubChem Compound in SMARTS format. Each SMARTS string is followed by a semicolon-separated incidence vector that specifies in how many records in which repository and with what radius the respective fragment was generated. As each, PubChem Substance and Compound, were fragmented with three radii in total $(r=1,2,3)$, the vector contains six values. Association between position in the vector, PubChem Substance/Compound, and atom environment radius is as follows: position 1) Substance $r=1$; position 2) Substance $r=2$; position 3) Substance $r=3$; position 4) Compound $r=1$; position 5) Compound $r=2$; position 6) Compound $r=3$. File is sorted by lexicographical order of SMARTS strings.

\section{Authors' contributions}

VDH devised the environment encoding, carried out the computations and analyzed the results, and drafted the manuscript. EEB facilitated the 
computations and edited the manuscript. SHB reviewed the final manuscript. All authors read and approved the final manuscript.

\section{Acknowledgements}

We thank the anonymous reviewers for their careful reading of our manuscript and their constructive comments, which helped us to improve the manuscript. This research was supported in part by the Intramural Research Program of the National Library of Medicine, National Institutes of Health, US Department of Health and Human Services.

\section{Compliance with ethical guidelines}

\section{Competing interests}

The authors declare that they have no competing interests.

Received: 29 January 2015 Accepted: 20 May 2015

Published online: 19 August 2015

\section{References}

1. Minkin VI (1951) Glossary of terms used in theoretical organic chemistry (IUPAC recommendations 1999). Pure Appl Chem 1999:71

2. Trudeau RJ (1993) Graphs. In Introduction to Graph Theory. Dover Publications, Inc., New York, p 19

3. Lewis GN (1916) The atom and the molecule. J Am Chem Soc 38:762-785

4. Cayley A (1874) On the mathematical theory of isomers. Philos Mag 47:444-447

5. Panico R, Powell WH, Richter JC (1993) A Guide to IUPAC Nomenclature of Organic Compounds Recommendations 1993. Blackwell Science, Oxford

6. Favre HA, Hellwich KH, Moss GP, Powell WH, Traynham JG (1999) Corrections to a guide to IUPAC nomenclature of organic compounds (IUPAC recommendations 1993). Pure Appl Chem 71:1327-1330

7. Leigh GJ, Favre HA, Metanomski WV (1998) Principles of organic nomenclature. Blackwell Science, Oxford

8. Skolnik H, Clow A (1964) A notation system for indexing pesticides. J Chem Doc 4:221-227

9. Dyson GM, Lynch MF, Morgan HL (1968) A modified IUPAC-Dyson notation system for chemical structures. Inform Storage Retr 4:27-83

10. Wiswesser WJ (1982) How the WLN began in 1949 and how it might be in 1999. J Chem Inf Comput Sci 22:88-93

11. Weininger D (1988) SMILES, a chemical language and information system. 1. Introduction to methodology and encoding rules. J Chem Inf Comput Sci 28:31-36

12. Weininger $D$, Weininger $A$, Weininger JL (1989) SMILES. 2. Algorithm for generation of unique SMILES notation. J Chem Inf Comput Sci 29:97-101

13. Barnard JM, Jochum CJ, Welford SM (1989) A universal structure/substructure representation for PC-host communication. In: Warr WA (ed) Chemical Structure Information Systems, ACS Symposium Series, vol 400. American Chemical Society, Washington DC, pp 76-81

14. Rohbeck HG (1991) Representation of structure description arranged linearly. In: Gmehlin J (ed) Software Development in Chemistry 5. Springer, Heidelberg, pp 49-58

15. Ash S, Cline MA, Homer RW, Hurst T, Smith GB (1997) SYBYL line notation (SLN): a versatile language for chemical structure representation. J Chem Inf Comput Sci 37:71-79

16. Homer RW, Swanson J, Jilek RJ, Hurst T, Clark RD (2008) SYBYL line notation (SLN): a single notation to represent chemical structures, queries, reactions, and virtual libraries. J Chem Inf Model 48:2294-2307

17. Gakh AA, Burnett MN (2001) Modular Chemical Descriptor Language (MCDL): composition, Connectivity, and Supplementary Modules. J Chem Inf Comput Sci 41:1494-1499

18. Gakh AA, Burnett MN, Trepalin SV, Yarkov AV (2011) Modular Chemical Descriptor Language (MCDL): stereochemical modules. J Cheminform 3:5

19. McNaught $A$ (2006) The IUPAC international chemical identifier: inChl-a new standard for molecular informatics. Chem Int 28:12-14

20. Heller SR, McNaught AD (2009) The IUPAC international chemical identifier. Chem Int 31:7-9
21. Proschak E, Wegner JK, Schüller A, Schneider G, Fechner U (2007) Molecular query language (MQL) - a context-free grammar for substructure matching. J Chem Inf Model 47:295-301

22. Reisen FH, Schneider G, Proschak E (2009) Reaction-MQL: line notation for functional transformation. J Chem Inf Model 49:6-12

23. Dalby A, Nourse JG, Hounshell WD, Gushurst AKI, Grier DL, Leland BA, Laufer J (1992) Description of several chemical structure file formats used by computer programs developed at Molecular Design Limited. J Chem Inf Comput Sci 32:244-255

24. (2011) Accelrys CTFile Formats. http://accelrys.com/products/informatics/cheminformatics/ctfile-formats/no-fee.php. Accessed 30 July 2015

25. (2005) TRIPOS Mol2 File Format. http://tripos.com/data/support/mol2. pdf. Accessed 30 July 2015

26. Warr WA (2011) Representation of chemical structures. Wiley Interdiscip Rev Comput Mol Sci 1:557-579

27. Carhart RE, Smith DH, Venkataraghavan R (1985) Atom pairs as molecular features in structure-activity studies: definition and applications. J Chem Inf Comput Sci 25:64-73

28. Sheridan RP, Miller MD, Underwood DJ, Kearsley SK (1996) Chemical similarity using geometric atom pair descriptors. J Chem Inf Comput Sci 36:128-136

29. Barnard JM, Downs GM (1997) Chemical fragment generation and clustering software. J Chem Inf Comput Sci 37:141-142

30. Filimonov D, Poroikov V, Borodina Y, Gloriozova T (1999) Chemical similarity assessment through multilevel neighborhoods of atoms: definition and comparison with the other descriptors. J Chem Inf Comput Sci 39:666-670

31. Durant JL, Leland BA, Henry DR, Nourse JG (2002) Reoptimization of MDL keys for use in drug discovery. J Chem Inf Comput Sci 42:1273-1280

32. Bender A, Mussa HY, Glen RC, Reiling S (2004) Molecular similarity searching using atom environments, information-based feature selection, and a naïve bayesian classifier. J Chem Inf Comput Sci 44:170-178

33. Bender A, Mussa HY, Glen RC, Reiling S (2004) Similarity searching of chemical databases using atom environment descriptors: evaluation of performance. J Chem Inf Comput Sci 44:1708-1718

34. Rogers D, Hahn M (2010) Extended-connectivity fingerprints. J Chem Inf Model 50:742-754

35. Barnard JM, Downs GM (1992) Clustering of chemical structures on the basis of two-dimensional similarity measures. J Chem Inf Comput Sci 32:644-649

36. Willett P (2000) Chemoinformatics — similarity and diversity in chemical libraries. Curr Opin Biotechnol 11:85-88

37. Brown RD, Martin YC (1996) Use of structure-activity data to compare structure-based clustering methods and descriptors for use in compound selection. J Chem Inf Comput Sci 36:572-584

38. McGregor MJ, Pallai PV (1997) Clustering of large databases of compounds using MDL "keys" as structural descriptors. J Chem Inf Comput Sci 37:443-448

39. MacCuish JD, MacCuish NE (2013) Chemoinformatics applications of cluster analysis. Wiley Interdiscip Rev Comput Mol Sci 4:34-48

40. Willett P (1998) Chemical similarity searching. J Chem Inf Comput Sci 38:983-996

41. Willett $P$ (2006) Similarity-based virtual screening using 2D fingerprints. Drug Discov Today 11:1046-1053

42. Willett $P$ (2011) Similarity searching using 2D structural fingerprints. Methods Mol Biol 672:133-158

43. Feldman A, Hodes $L$ (1975) An efficient design for chemcial structure searching. I. The screens. J Chem Inf Comput Sci 15:147-152

44. Xiao Y, Qiao Y, Zhang J, Lin S, Zhang W (1997) A method for substructure search by atom-centered multilayer code. J Chem Inf Comput Sci 37:701-704

45. Liu P, Agrafiotis DK, Rassokhin DN (2001) Power Keys: a novel class of topological descriptors based on exhaustive subgraph enumeration and their application in substructure searching. J Chem Inf Model 51:2843-2851

46. Crowe JE, Lynch MF, Town WG (1970) Analysis of structural characteristics of chemical compounds in a large computer-based file. Part I. Non-cyclic fragments. J Chem Soc C 990-996. doi:10.1039/J39700000990

47. Adamson GW, Lynch MF, Town WG (1971) Analysis of structural characteristics of chemical compounds in a large computer-based File. Part 
II. Atom-centred fragments. J Chem Soc C 3702-3706. doi:10.1039/ J39710003702

48. Larsen PO, von Ins M (2010) The rate of growth in scientific publication and the decline in coverage provided by Science Citation Index. Scientometrics 84:575-603

49. Binetti R, Costamagna FM, Marcello I (2008) Exponential growth of new chemicals and evolution of information relevant to risk control. Ann Ist Super Sanita 44:13-15

50. Chemical Abstracts Service (2008) CAS Statistical Summary 1907-2007. Chemical Abstracts Service, Columbus $(\mathrm{OH})$

51. Bolton EE, Wang Y, Thiessen PA, Bryant SH (2008) Chapter 12 PubChem: integrated platform of small molecules and biological activities. In: Wheeler RA, Spellmeyer DC (eds) Annual reports in computational chemistry, vol 4. Elsevier, Oxford, pp 217-241

52. Wang Y, Xiao J, SuzekTO, Zhang J, Wang J, Zhou Z et al (2012) PubChem's BioAssay database. Nucleid Acids Res 40:D400-D412

53. Wang YL, Bolton E, Dracheva S, Karapetyan K, Shoemaker BA, Suzek TO et al (2010) An overview of the PubChem BioAssay resource. Nucleic Acids Res 38:D255-D266

54. (2004) The PubChem Project. http://pubchem.ncbi.nlm.nih.gov/. Accessed 30 July 2015

55. Petitjean M (1992) Applications of the radius-diameter diagram to the classification of topological and geometrical shapes of chemical compounds. J Chem Inf Comput Sci 32:331-337

56. (2015) RDKit: Open-Source Cheminformatics Software. http://www.rdkit. org. Accessed 30 July 2015

57. (2014) OpenEye OEChem C++ Toolkit, version 2.0.3.b.1. OpenEye Scientific Software, Inc., Santa Fe (NM). http://www.eyesopen.com/oechem-tk. Accessed 30 July 2015

58. Hückel E (1931) Quantentheoretische Beiträge zum Benzolproblem I. Die Elektronenkonfiguration des Benzols und verwandter Verbindungen. Z Phys 70:204-286

59. Hückel E (1932) Quantentheoretische Beiträge zum Benzolproblem II. Quantentheorie der induzierten Polaritäten. Z Phys 72:310-337

60. OpenEye Scientific Software, Inc. (2012) OEChem C++ Toolkit v1.9.2 Manual. OpenEye Scientific Software, Inc., Santa Fe, p 50

61. Claassen HH, Selig H, Malm JG (1962) Xenon Tetrafluoride. J Am Chem Soc 84:3593

62. MacKenzie DR (1963) Krypton Difluoride: preparation and handling. Science 141:1171

63. Templeton DH, Zalkin A, Forrester JD, Williamson SM (1963) Crystal and molecular structure of xenon trioxide. J Am Chem Soc 85:817

64. Selig H, Malm JG, Claassen HH, Chernick CL, Huston JL (1964) Xenon tetroxide-preparation and some properties. Science 143:1322-1323

65. Graham L, Graudejus O, Jha NK, Bartlett N (2000) Concerning the nature of XePtF6. Coord Chem Rev 197:321-334

66. Khriachtchev L, Pettersson M, Runeberg N, Lundell J, Räsänen M (2000) A stable argon compound. Nature 406:874-876

67. Tramšek M, Žemva B (2006) Synthesis, properties and chemistry of xenon(II) fluoride. Acta Chim Slov 53:105-116
68. Heifets A, Jurisica I (2012) SCRIPDB: a portal for easy access to syntheses, chemicals and reactions in patents. Nucl Acids Res 40:D428-D433

69. (2011) SCRIPDB. University of Toronto. http://dcv.uhnres.utoronto.ca/ SCRIPDB/. Accessed 30 July 2015

70. IBM Almaden Research Center, 650 Harry Road, San Jose, CA 95120 (USA)

71. (2007) ChemSpider. http://www.chemspider.com/. Accessed 30 July 2015

72. (2009) PubChem Substructure Fingerprint V1.3, National Center for Biotechnology Information, Bethesda. ftp://ftp.ncbi.nlm.nih.gov/pubchem/ specifications/pubchem_fingerprints.txt. Accessed 30 July 2015

73. de Silva KM, Goodman JM (2005) What is the smallest saturated acyclic alkane that cannot be made? J Chem Inf Model 45:81-87

74. Paton RS, Goodman JM (2007) Exploration of the accessible chemical space of acyclic alkanes. J Chem Inf Model 47:2124-2132

75. Kolodzik A, Urbaczek S, Rarey M (2012) Unique ring families: a chemically meaningful description of molecular ring topologies. J Chem Inf Model 52:2013-2021

76. Ertl P, Schuffenhauer A (2009) Estimation of synthetic accessibility score of drug-like molecules based on molecular complexity and fragment contributions. J Cheminform 1:8

77. Daylight Theory Manual, Chapter 4: SMARTS—A Language for Describing Molecular Patterns. Daylight Chemical Information Systems, Inc., Laguna Niguel http://www.daylight.com/dayhtml/doc/theory/theory.smarts. html. Accessed Sep 2013

78. OpenEye Lexichem C++ Toolkit. OpenEye Scientific Software, Inc., Santa Fe. http://www.eyesopen.com/lexichem-tk. Accessed 30 July 2015

79. Schomburg K, Ehrlich HC, Stierand K, Rarey M (2010) From structure diagrams to visual chemical patterns. J Chem Inf Model 50:1529-1535

80. (2010) SMARTSviewer. Center for Bioinformatics, Universität Hamburg. http://smartsview.zbh.uni-hamburg.de/. Accessed 30 July 2015

\section{Publish with ChemistryCentral and every scientist can read your work free of charge \\ "Open access provides opportunities to our colleagues in other parts of the globe, by allowing anyone to view the content free of charge."}

W. Jeffery Hurst, The Hershey Company.

- available free of charge to the entire scientific community

- peer reviewed and published immediately upon acceptance

- cited in PubMed and archived on PubMed Central

- yours - you keep the copyright

Submit your manuscript here:

http://www.chemistrycentral.com/manuscript/ 